\title{
Cardiolipin-Dependent Mitophagy Guides Outcome after Traumatic Brain Injury
}

\author{
Honglu Chao, ${ }^{1,3,4 \star}$ Chao Lin, ${ }^{1 \star}$ Qiang Zuo, ${ }^{2 \star}$ Yinlong Liu, ${ }^{1}$ Mengqing Xiao,,${ }^{5,6,7}$ Xiupeng Xu, ${ }^{1}$ Zheng Li, ${ }^{1}$ Zhongyuan Bao, ${ }^{1}$ \\ Huimei Chen, ${ }^{8,9}$ Yongping You, ${ }^{1}$-Patrick M. Kochanek, ${ }^{4}$-Huiyong Yin, ${ }^{5,6,7,10}$ Ning Liu, ${ }^{1}$ Valerian E. Kagan, ${ }^{3,11}$ \\ Hülya Bayır, ${ }^{3,4,12}$ and Jing Ji $\mathbf{i}^{1,3,4}$ \\ Departments of ${ }^{1}$ Neurosurgery and ${ }^{2}$ Orthopedics, the First Affiliated Hospital of Nanjing Medical University, Nanjing 210029 , China, ${ }^{3}$ Center for Free \\ Radical and Antioxidant Health, Department of Environmental and Occupational Health, ${ }^{4}$ Safar Center for Resuscitation Research, Department of Critical \\ Care Medicine, University of Pittsburgh, Pittsburgh, Pennsylvania 15219, ${ }^{5}$ Key Laboratory of Food Safety Research, Institute for Nutritional Sciences, \\ Shanghai Institutes for Biological Sciences, Chinese Academy of Sciences (CAS), Shanghai 200031, China, ${ }^{6}$ University of the Chinese Academy of Sciences, \\ CAS, Beijing 100049, China, ${ }^{7}$ School of Life Science and Technology, Shanghai Tech University, Shanghai 201210, China, ${ }^{8}$ Department of Medical Genetics, \\ Nanjing University School of Medicine, Nanjing 210093, China, ${ }^{9}$ Jiangsu Key Laboratory of Molecular Medicine, Nanjing 210029, China, ${ }^{10}$ Key Laboratory of \\ Food Safety Risk Assessment, Ministry of Health, Beijing 100022, China, ${ }^{11}$ Laboratory of Navigational Redox Lipidomics and Department of Human \\ Pathology, IM Sechenov Moscow State Medical University, Moscow 119991, Russian Federation, and ${ }^{12}$ Children's Neuroscience Institute, Children's \\ Hospital of Pittsburgh, Pittsburgh, Pennsylvania 15224
}

Mitochondrial energy production is essential for normal brain function. Traumatic brain injury (TBI) increases brain energy demands, results in the activation of mitochondrial respiration, associated with enhanced generation of reactive oxygen species. This chain of events triggers neuronal apoptosis via oxidation of a mitochondria-specific phospholipid, cardiolipin (CL). One pathway through which cells can avoid apoptosis is via elimination of damaged mitochondria by mitophagy. Previously, we showed that externalization of CL to the mitochondrial surface acts as an elimination signal in cells. Whether CL-mediated mitophagy occurs in vivo or its significance in the disease processes are not known. In this study, we showed that TBI leads to increased mitophagy in the human brain, which was also detected using TBI models in male rats. Knockdown of CL synthase, responsible for de novo synthesis of CL, or phospholipid scramblase-3, responsible for CL translocation to the outer mitochondrial membrane, significantly decreased TBI-induced mitophagy. Inhibition of mitochondrial clearance by 3-methyladenine, mdivi-1, or phospholipid scramblase-3 knockdown after TBI led to a worse outcome, suggesting that mitophagy is beneficial. Together, our findings indicate that TBI-induced mitophagy is an endogenous neuroprotective process that is directed by CL, which marks damaged mitochondria for elimination, thereby limiting neuronal death and behavioral deficits.

Key words: apoptosis; autophagy; cardiolipin; mitophagy; neuroprotection; phospholipid

\section{Significance Statement}

Traumatic brain injury (TBI) increases energy demands leading to activation of mitochondrial respiration associated with enhanced generation of reactive oxygen species and resultant damage to mitochondria. We demonstrate that the complete elimination of irreparably damaged organelles via mitophagy is activated as an early response to TBI. This response includes translocation of mitochondria phospholipid cardiolipin from the inner membrane to the outer membrane where externalized cardiolipin mediates targeted protein light chain 3-mediated autophagy of damaged mitochondria. Our data on targeting phospholipid scramblase and cardiolipin synthase in genetically manipulated cells and animals strongly support the essential role of cardiolipin externalization mechanisms in the endogenous reparative plasticity of injured brain cells. Furthermore, successful execution and completion of mitophagy is beneficial in the context of preservation of cognitive functions after TBI.

\section{Introduction}

The bioenergetics of the brain relies mainly on the respiratory oxidation of glucose, thus making mitochondria essential for the maintenance of the brain's normal function. Aberrant mitochondrial dynamics and metabolism play an important role in the 
pathogenesis of a number of neurological diseases (Zhang et al., 2013; Pickrell and Youle, 2015).

TBI leads to an acute energy crisis that sharply raises the demands of mitochondrial electron transport (Kilbaugh et al., 2015). This is mostly because of the rupture of membrane barriers and ionic dishomeostasis triggering immediate compensatory attempts to reinstate the balance by "re-pumping" the ions back into their respective compartments at the expense of ATP hydrolysis (Liu et al., 2013). These attempts lead to the increased respiration inevitably associated with the enhanced production of reactive oxygen species.

Our previous work demonstrated that this chain of events culminates in apoptosis of neurons realized via specific oxidation of a mitochondria-unique phospholipid, CL, catalyzed by the intermembrane hemoprotein cytochrome $c$ (cyt $c$; Kagan et al., 2005; Ji et al., 2012b). Among the pathways neurons employ to avoid this vicious cycle and prevent apoptosis is effective elimination of damaged mitochondria through a specialized autophagic process, mitophagy. We reported that CL is used by cells as a signal for mitophagy via its translocation to the mitochondrial surface, where it is specifically recognized by the autophagic protein microtubuleassociated protein light chain 3 (LC3; Chu et al., 2013, 2014). Although the utility of CL mitophagic signaling has been demonstrated in neuronal cells in vitro, the occurrence and role of CL-mediated mitophagy in vivo, particularly in regulation of the pathogenic processes in disease, remain to be defined and could be important in the context of new drug discovery for TBI.

In the present study, we documented that TBI is associated with increased evidence of mitophagy in the human brain. In parallel preclinical studies, using the model of controlled cortical impact (CCI) on rat brain and in vitro model of neuronal mechanical stretch, we established that mitophagy is dependent on the externalization of CL on the surface of mitochondria. We further showed that suppressed clearance of injured mitochondria leads to the worsened CCI outcome. Together, our data suggest that TBIinduced mitophagy, operating via CL externalization as markers for the elimination of damaged mitochondria, is beneficial because it attenuates neuronal death and associated behavioral deficits.

\section{Materials and Methods}

Patients. Human brain tissue was obtained for analysis from the tissue bank of the Institutional Review Board-approved Department of Neurosurgery at the First Affiliated Hospital of Nanjing Medical University (Table 1). Brain tissue resected from the pericontusional area of the temporal or frontal cortices of severe TBI patients who underwent decompressive craniectomy was flash-frozen in liquid nitrogen and stored at $-80^{\circ} \mathrm{C}$ until analysis. Brain tissue resected from the temporal cortices of epilepsy patients who underwent surgical treatment was used as a control and stored in the same manner as the TBI samples.

Rat TBI model. All research protocols and animal experiments were approved by Nanjing Medical University according to the guidelines of the Animal Care and Use Committee. Briefly, a craniotomy was performed over the left parietal cortex in 17-d-old male Sprague-Dawley rats. Rats were anesthetized with 3.5\% isoflurane in $\mathrm{O}_{2}$ and maintained on $2 \%$ isoflurane in $\mathrm{N}_{2} \mathrm{O} / \mathrm{O}_{2}(2: 1)$. For all experiments, a pneumatically driven, $6 \mathrm{~mm}$ metal impactor tip was used at a velocity of $4.0 \pm 0.2 \mathrm{~m} / \mathrm{s}$,

This work was supported by NIH Grants (NS061817, NS076511) to H.B., National Natural Science Foundation of China $(81300998,81471269)$ to J.J., Jiangsu Province's Natural Science Foundation (BK20160047, BK20160044) to J.J., and Jiangsu Province and the Priority Academic Program Development of Jiangsu Higher Education Institutions. The authors declare no competing financial interests.

*H. Chao, C.L., and Q.Z. contributed equally to this work.

Correspondence should be addressed to Hülya Bayır at bayihx@ccm.upmc.edu or Jing Ji at jijing@njmu.edu.cn. https://doi.org/10.1523/JNEUROSCI.3415-17.2018

Copyright $\odot 2019$ the authors $\quad 0270-6474 / 19 / 391931-14 \$ 15.00 / 0$
Table 1. Clinical information of human brain specimens

\begin{tabular}{|c|c|c|c|c|c|c|}
\hline $\begin{array}{l}\text { No. of } \\
\text { patients }\end{array}$ & Gender & Age, y & Diagnosis & $\begin{array}{l}\text { Time to } \\
\text { injury, h }\end{array}$ & Site & Outcome \\
\hline \multicolumn{7}{|l|}{$\mathrm{Ctrl}$} \\
\hline 1 & Male & 52 & Epilepsy & - & Right temporal lobe & No disability \\
\hline 2 & Male & 21 & Epilepsy & - & Right temporal lobe & No disability \\
\hline 3 & Male & 58 & Epilepsy & - & Left temporal lobe & Mild disability \\
\hline \multicolumn{7}{|l|}{ TBI } \\
\hline 1 & Male & 54 & TBI & 3 & Left frontal lobe & Severe disability \\
\hline 2 & Male & 68 & TBI & 5 & Left frontal lobe & Death \\
\hline 3 & Male & 61 & TBI & 8 & Right frontal lobe & Severe disability \\
\hline 4 & Male & 62 & TBI & 3 & Right temporal lobe & Death \\
\hline 5 & Male & 56 & TBI & 6 & Left temporal lobe & Mild disability \\
\hline
\end{tabular}

at a penetration depth of $2.5 \mathrm{~mm}$, for a duration of $50 \mathrm{~ms}$. After TBI, the bone flap was replaced, sealed with dental cement, and the scalp incision was closed. The rats were weaned from mechanical ventilation, extubated, and returned to their cages until euthanasia. The basic surgical preparation and procedure were performed in accordance with previously described methods (Lai et al., 2008; Ji et al., 2012b).

All animal experiments were performed in accordance with the NIH Guidelines for the Care and Use of Animals and approved by the Animal Care and Use Committee of Nanjing Medical University in China and the University of Pittsburgh.

Primary cortical neuronal cultures and an in vitro TBI model. Cortices were isolated from embryonic Day 17 Sprague-Dawley rats in an ice-cold medium. Briefly, the tissue was rinsed and triturated to obtain the primary neuron suspension. Primary cortical neurons were plated at a density of $3.5 \mathrm{ml} /$ well $\left(2.0 \times 10^{6} \mathrm{cells} / \mathrm{ml}\right)$ and were used to perform experiments at $7 \mathrm{~d}$ in vitro, according to the study protocol. TBI was produced via mechanical stretch of the neurons cultured on a flexible SILASTIC membrane using a pneumatic pressure pulse as described previously (Ji et al., 2012a).

GFP-LC3 transgenic rats. GFP-LC3 transgenic rats were purchased from Beijing CasGene Biotech. Co., Ltd. After kill, brains were perfused with $4 \%$ paraformaldehyde, followed by dehydration at increasing concentrations of sucrose (10-30\%). Selected areas of the cortex and hippocampus were processed as $5-\mu \mathrm{m}$-thick cryosections that were dried at room temperature and stained with mitochondria markers. Sections were observed under a light laser confocal microscope and analyzed using Image Pro-Plus 7.0 software.

Drug administration. To test the in vivo effect of different drugs after TBI, injured animals were randomly assigned to different groups receiving either vehicle (DMSO) or one of the other drugs being tested. Five microliters of 3-methyladenine (3-MA; $10 \mathrm{mg} / \mathrm{ml}$, diluted in DMSO; sc-205596, Santa Cruz Biotechnology) or bafilomycin A1 (BAF-A1; 0.1 $\mathrm{mg} / \mathrm{ml}$, diluted in DMSO; sc-201550, Santa Cruz Biotechnology) was administered by a single intracerebroventricular injection $30 \mathrm{~min}$ before CCI. Mdivi-1 (1.5 mg/kg of $20 \mathrm{mg} / \mathrm{ml}$, diluted in DMSO; sc-215291, Santa Cruz Biotechnology) was administered to rats by intraperitoneal injection $15 \mathrm{~min}$ before CCI.

SiRNA administration. For in vivo administration, $30 \mathrm{nmol}$ of siRNA was infused through a surgically implanted brain infusion cannula (coordinates: $-0.8 \mathrm{~mm}$ posterior to bregma, $-1.5 \mathrm{~mm}$ lateral to midline, and $-4.6 \mathrm{~mm}$ ventral to the skull surface) using an osmotic minipump (Alzet Model 1003D, Durect) at a rate of $1.0 \mu \mathrm{l} / \mathrm{d}$ over $72 \mathrm{~h}$. The siRNA targeting rat CL synthase (CLS) and phospholipid scramblase-3 (PLS3), as well as the negative control siRNA, were synthesized by GenePharm (GENEPHARM). The efficacy of the knockdown of targeted proteins was evaluated by Western blot. Primary neurons were transfected on Day 4 with $45 \mathrm{nmol}$ of targeted siRNA or control siRNA using Lipofectamine 3000 (Invitrogen). The knockdown efficacy was assessed by Western blot. Experiments were performed $72 \mathrm{~h}$ after transfection.

Mitochondrial cyt c release. Samples (pericontusional area in rat brain after CCI) were washed in PBS and then incubated for $3 \mathrm{~min}$ in lysis buffer ( $75 \mathrm{~mm} \mathrm{NaCl}, 8 \mathrm{~mm} \mathrm{Na}_{2} \mathrm{HPO}_{4}, 1 \mathrm{~mm} \mathrm{NaH} \mathrm{PO}_{4}, 1 \mathrm{~mm}$ EDTA, and $350 \mu \mathrm{g} / \mathrm{ml}$ digitonin). The lysates were centrifuged at $12,000 \times g$ for $1 \mathrm{~min}$ and the 
supernatant was collected for cytosolic cyt $c$ analysis. The mitochondriaenriched fraction was resuspended in lysis buffer. The suspension was then incubated on ice and sonicated twice for $10 \mathrm{~s}$ with a $30 \mathrm{~s}$ interval. The final mitochondrial lysate was once again centrifuged at 400,000 $\times g$ in an ultracentrifuge (Backman) for $25 \mathrm{~min}$ and the supernatant collected for mitochondrial cyt $c$ analysis. Cyt $c$ was detected by Western blot.

Transmission electron microscopy. To collect samples for microscopic examination, rats were killed using $10 \%$ chloral hydrate and perfused with $4 \%$ paraformaldehyde. The brain was extracted and cortices and hippocampi were isolated in ice-cold PBS. Brain tissue was fixed in PBS, $\mathrm{pH} 7.4$, containing $2.5 \%$ glutaraldehyde for at least $2 \mathrm{~h}$ at room temperature. After this step, both rat tissue and prefixed human tissue samples were processed in the same way. Tissues were postfixed in $1 \%$ osmium tetroxide for $1 \mathrm{~h}$ at $4^{\circ} \mathrm{C}$ and dehydrated with an ethanol gradient, followed by embedding in epoxy resin. The ultrastructure of the tissue (100 $\mathrm{nm}$ ultrathin sections) was observed using a transmission electron microscope (Quanta 10, FEI). The autophagic vacuoles containing damaged mitochondria were quantified by an investigator blinded to group assignment from 20 fields-of-view at $4800 \times$ direct magnification. Image analysis was performed using ImageJ (Schneider et al., 2012).

Confocal microscopy. Colocalization of the mitochondrial marker (MitoTracker Red) with GFP-LC3 was performed to ascertain the occurrence of mitophagy in accordance with previously described guidelines (Klionsky et al., 2016). Primary cortical neurons were transfected with an adenovirus of GFP-LC3 to visualize LC3 puncta, followed by staining with MitoTracker Red to visualize mitochondria under Lipofectamine 2000 (Invitrogen). GFP-LC3 transgenic rats were used to assess CCIinduced mitophagy in vivo. Fluorescence images were captured and viewed using a light laser confocal microscope. Multiple fields from one coverslip/well were selected to get an average at random and imaged for further analysis using Image Pro-Plus 7.0 software. Each experiment was repeated independently in triplicate or more. The following primary antibodies were used: Neu-N (1:1000; Abcam, catalog \#ab104224), COXIV (1:200; Abcam, catalog \#ab16056).

Immunoblot analysis. Extracts for immunoblotting were obtained from brain tissues and cells homogenized in RIPA buffer. In brief, proteins were separated by electrophoresis and then transferred to PVDF membranes by electro blotting, as described in standard immunoblot procedures. The primary antibodies were p62 (1:1000; Abcam, catalog \#ab56416; RRID:AB_945626), LC3 (1:1000; Cell Signaling Technology, catalog \#2775s), TOM40 (1:1000; Abcam, catalog \#ab51884; RRID: AB_883141), COXIV (1:1000; Cell Signaling Technology, catalog \#11967), MnSOD (1:1000; Cell Signaling Technology, catalog \#13141), GAPDH (1: 1000; Cell Signaling Technology, catalog \#5174; RRID:AB_10622025), CLS (1:1000; Abcam, catalog \#ab156882), PLS3 (1:1000; Abcam, catalog \#ab137128), and Cyt c (1:1000; Abcam, catalog \#ab13575; RRID: AB_300470).

Assessment of CL externalized to the mitochondrial surface using flow cytometry. As previously described, an annexin V-binding assay was used to assess the amount of CL on the surface of mitochondria (Kagan et al., 2016). In brief, MitoTracker Red CMXRos (Life Technologies) was used to label mitochondria before harvesting. Isolated crude mitochondria were incubated with FITC-labeled annexin V to stain surface-exposed CL and then subjected to flow cytometry analysis (FACSCanto, Becton Dickinson). The FITC fluorescence from gated red fluorescent mitochondria (MitoTracker Red) events was determined to evaluate the binding of annexin $\mathrm{V}$ to mitochondria. Data presented are the relative FITC fluorescence intensity compared with that of mitochondria isolated from control brains.

TUNEL. A terminal deoxynucleotidyl transferase dUTP nick-end labeling (TUNEL) assay was used to assess apoptotic cell death according to the manufacturer's instructions (12156792910, Roche). The rats were killed and perfused with $0.1 \mathrm{~mm}$ PBS, $\mathrm{pH}$ 7.4. In brief, coronal frozen sections were embedded onto the slides and incubated with $50 \mu \mathrm{l}$ TUNEL reaction mixture. The slides were observed and quantified using a Nikon fluorescent microscope. The apoptotic cells showed red fluorescence. The nuclei were stained with DAPI and showed blue fluorescence. Apoptosis was calculated as TUNEL-positive cells/DAPI.

Cortical lesion volume. Lesion size was assessed on Day 7 post-CCI. One section was obtained every $0.5 \mathrm{~mm}$ through dorsal hippocampus for lesion volume measurement. Free-floating sections were washed three times in PBS. After blocking in 5\% serum and $0.25 \%$ Triton X-100 in PBS for $60 \mathrm{~min}$, the sections were incubated in blocking solution containing anti-NeuN antibody (1:1500; ab177487, Abcam) at $4^{\circ} \mathrm{C}$ overnight. After this primary antibody incubation, the sections were washed and incubated with a secondary anti-rabbit antibody for $60 \mathrm{~min}$ in blocking solution. Visualization was performed using a Vectorstain ABC kit (PK-6100, Vector Laboratories). Stained sections were mounted on glass slides. Necrotic divot and contralateral cortical areas and volumes were calculated by the Image analysis software. The area representing ipsilateral lesioned and contralateral healthy cortex was first calculated per section by multiplying the number of points counted per region. The volume (V) was estimated by multiplying the sum of areas $(\sigma \mathrm{A})$ by the periodicity $(m)$ and the mean section thickness $(t): \mathrm{V}=(\sigma \mathrm{A}) m t$. The cortical lesion volume in the ipsilateral cortex is expressed as a percentage of the volume of the entire contralateral cortex volume by the equation: $\% \mathrm{~V}=$ (Vdivot/ Vcontra) $\times 100$ (Whalen et al., 1999; Scafidi et al., 2010).

Assessments of motor function and cognitive performance. Motor function was evaluated using beam balance, which was performed on postinjury Days $1-5$ by the same experienced investigator blinded to the experimental group assignment. The beam balance test was used to assess gross vestibular-motor function. This test consists of placing the animal on a suspended, narrow wooden beam, and the animals are pretrained so that each animal can stand on the beam for $60 \mathrm{~s}$ before CCI. The amount of time for which rats could remain (in seconds) on the beam balance was recorded and analyzed using Prism Software 6.04 (GraphPad Software).

For the Morris water maze (MWM), the water was colored black and the temperature was maintained at $25 \pm 2^{\circ} \mathrm{C}$. To ensure recovery from motor deficits, hidden platform testing was performed on post-TBI Days $11-15$, and visible platform testing was performed on Days 16-17. Rats were allowed a maximum of $120 \mathrm{~s}$ to look for the submerged platform. If rats failed to reach the platform by $120 \mathrm{~s}$, the experimenter placed them on the platform for $15 \mathrm{~s}$. There was a minimum of 5 min between each trial. The latencies for rats to reach the platform were recorded and analyzed using tracking device and software (Chromotrack 3.0, San Diego Instruments).

In a separate group of rats, assessments of neurocognitive function were performed using a NOR test on Days 18-19 after sham or CCI injury. Briefly, for the habituation phase, which was conducted $18 \mathrm{~d}$ after CCI injury; two similar objects were placed into the inner arena across from where the rat was placed. The rats were allowed to investigate for 35 min to become familiar with the objects. Exploration was defined as sniffing and/or touching the objects. The rats were returned to their home cages at the end of the habituation period, and $24 \mathrm{~h}$ later (that is, post-injury Day 19) were again placed in the arena with the objects for 1 min before being returned briefly to their home cages. The recognition test was conducted $1 \mathrm{~h}$ later and consisted of one of the familiar objects being replaced with a novel object, followed by recording the time spent exploring the original versus novel object.

Fluoro jade-C staining. Fluoro jade-C (FJC) was purchased from Sigma-Aldrich (catalog \#AG325) and staining was performed as described previously (Shellington et al., 2011). Briefly, the slide-mounted tissue was stained with $0.001 \%$ FJC solution and dried in an oven in the dark for over $1 \mathrm{~h}$. The dried tissue sections were then cleared by immersion in xylene for at least $1 \mathrm{~min}$, covered in neutral gum, and coverslipped. The slides were imaged under a fluorescent microscope. Nuclei were stained with DAPI and showed blue fluorescence. FJC-positive cells (green signal) were counted from five randomly chosen fields and the average number of cells was calculated.

Caspase activity. Caspase- 3 activity was quantified using a luminescence Caspase-Glo assay kit according to the manufacturer's instructions (Promega). Brain samples were transferred into ice-cold Tris-HCl buffer solution. Samples were collected at $15,000 \times g$ at $4^{\circ} \mathrm{C}$ for $10 \mathrm{~min}$. The supernatant was assayed using Assay kit. Luminescence was established at the baseline and following $1 \mathrm{~h}$ of incubation at RT with a Fusion-a plate reader (Perkline). Caspase-3 activity was indicated as the luminescence generated after $1 \mathrm{~h}$ of incubation per milligram of protein.

Statistical analysis. Results are reported as the mean \pm SD for immunoblots and fluorescence experiments. Gray levels were detected with 
A

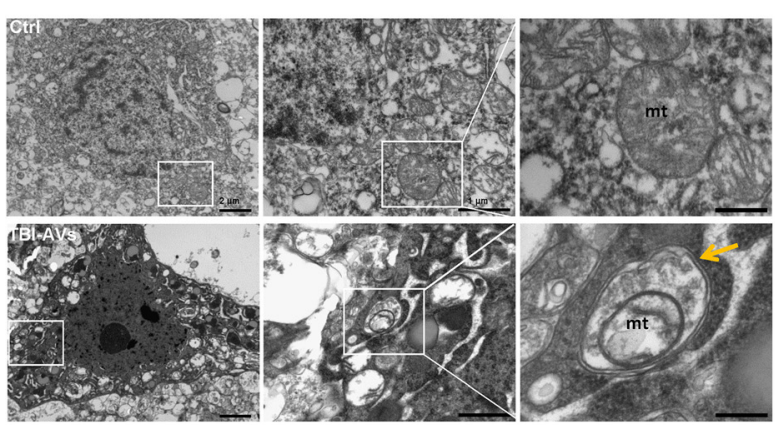

E
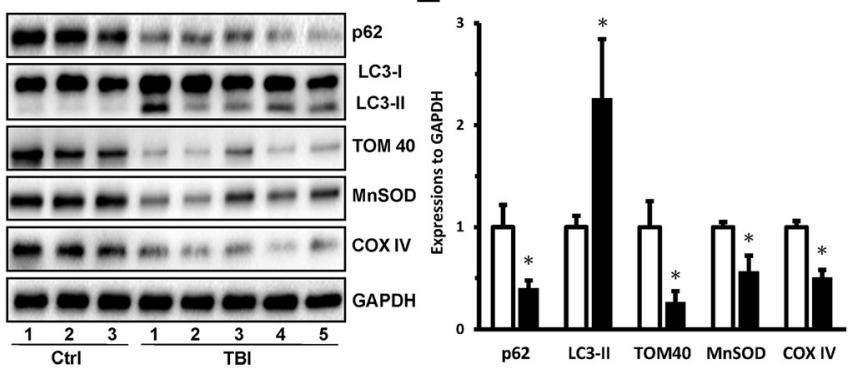

B

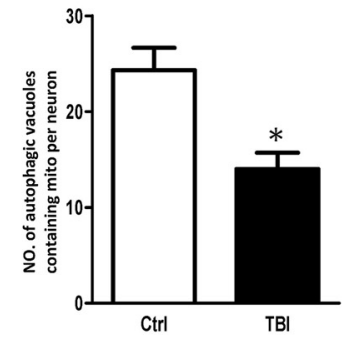

C

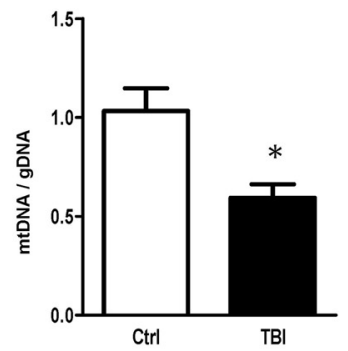

\section{F}

G

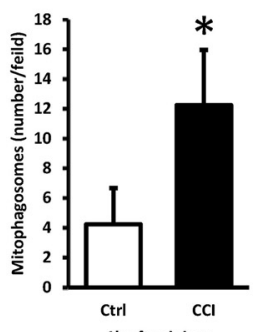

J

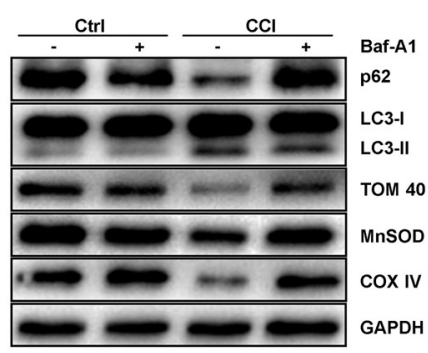

H

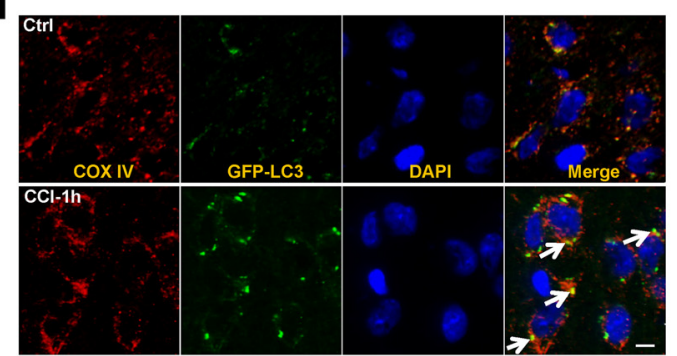

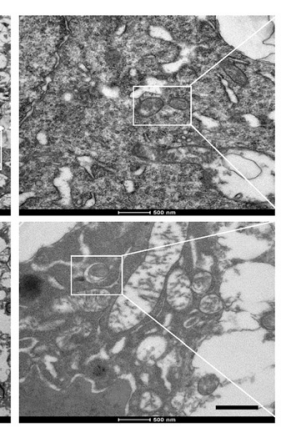

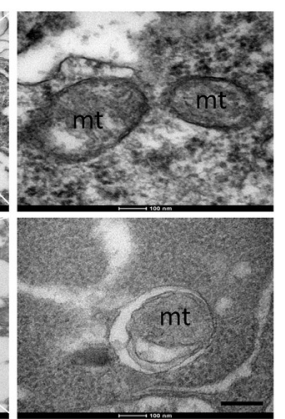

I

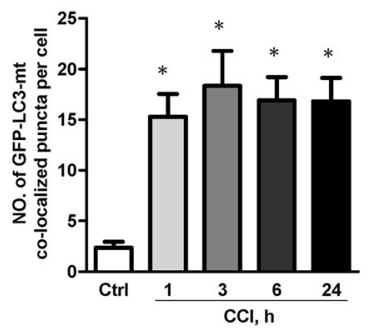

K

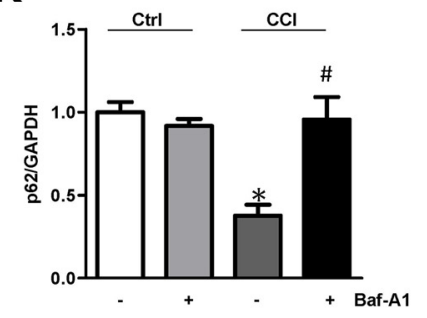

L

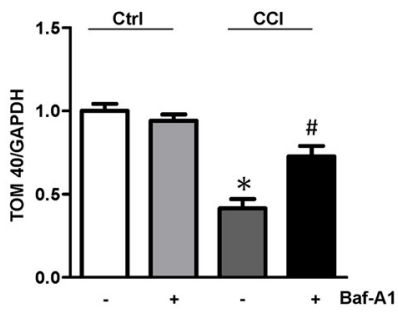

Figure 1. Mitophagy induction after traumatic brain injury. $A, B$, Representative TEM images of neurons from human brains. Mitochondria within autophagosome-like structures, such as a double membrane (arrow), were observed in TBI brain tissue. A significant difference in the number of autophagic vacuoles containing mitochondria per neuron (obtained from TEM images) was observed between $\mathrm{TBI}(n=5)$ and $\operatorname{Ctrl}\left(\mathrm{Ctrl} n=3, \mathrm{TBI} n=5 ;\right.$ mean $\pm \mathrm{SD} ; t=6.723 ;{ }^{*} p<0.05 \mathrm{vs}(\mathrm{trl}$ by $t$ test). $C$, The ratio of $\mathrm{mtDNA} / \mathrm{gDNA}$ was assessed by real-time $\mathrm{PCR}$, reflecting the relative number of mitochondria in human samples (Ctrl $n=3, \mathrm{TBI} n=5$, mean $\pm \mathrm{SD} ; t=8.534 ;{ }^{*} p<0.05 \mathrm{vs}$ (trl by $t$ test). $\boldsymbol{D}$, $\boldsymbol{E}$, Western blot of autophagic and mitochondrial markers obtained from TBI $(n=5)$ and control $(n=3)$ patients. GAPDH was used as the loading control. Bar graphs show the results of analysis (by band-density analysis) of p62, LC3-II, TOM40, MnSOD, and COXIV (Ctrl $n=3, \mathrm{TBI} n=5$, mean \pm SD; p62 $t=3.325$, LC3-II $t=7.364$, TOM40 $t=4.127, \mathrm{MnSOD} t=2.633$, COXIV $t=3.172 ;{ }^{*} p<0.05$ vs (trl by $t$ test). $\boldsymbol{F}, \mathbf{G}$, TEM images of neurons in the rat brains $1 \mathrm{~h}$ after CCl; note that mitochondria were engulfed by autophagic vacuoles. Scale bar, $0.1 \mu \mathrm{m}$. Autophagic vacuoles containing damaged mitochondria were quantified after $\mathrm{CCI}(\mathrm{Ctrl} n=3, \mathrm{TBI} n=$ 5 ; mean $\pm S D ; t=4.571 ;{ }^{*} p<0.05$ vs (trl by t test). $\boldsymbol{H}, \mathbf{I}$, Detection of mitophagy in the injured cortex of the rat after CCI (in vivo TBI model). CCl increased colocalization of GFP-LC3 and mitochondria (arrows) versus control $\left(n=5\right.$; mean $\pm S D ; F_{(4,20)}=20.317 ;{ }^{*} p<0.05$ vs Ctrl by one-way ANOVA and Tukey's posthoc test). Scale bar, $10 \mu$ m. $J$ - $L$, CCl-induced decrease of mitochondrial markers (TOM40, MnSOD, and COXIV) was partially reversed by Baf-A1-mediated inhibition of autophagosomal-lysosomal degradation; GAPDH was set as the standard $\left(n=3 ;\right.$ mean \pm SD; $\mathrm{p} 62: F_{(4,8)}=$ 9.532,TOM40: $F_{(4,8)}=7.332 ;{ }^{*} p<0.05$ vs control; \#p $<0.05$ vs CCl by one-way ANOVA and Tukey's post hoc test). Baf-A1, Bafilomycin A1; mt, mitochondria; TBI, traumatic brain injury; CCl, controlled cortical impact; TBI-AVs, autophagic vacuoles; h, hour; TEM, Transmission electron microscopy; Ctrl, control group.

ImageJ. Student's $t$ test was used to compare the differences between the two groups with Prism Software 6.04 (GraphPad Software). For behavioral tests, data were expressed as the mean \pm SEM. Motor and MWM data, which are continuous, were analyzed by two-way ANOVA for overall statistical significance, followed by Tukey's post hoc test for betweengroup comparisons. Significant differences were defined as $p<0.05$.

\section{Results}

Mitophagy is induced after clinical and experimental TBI Although general markers of autophagy have been shown to increase in the brain after experimental and clinical TBI (Clark et al., 2008; Cavallucci et al., 2014; Sarkar et al., 2014), the selective 
A

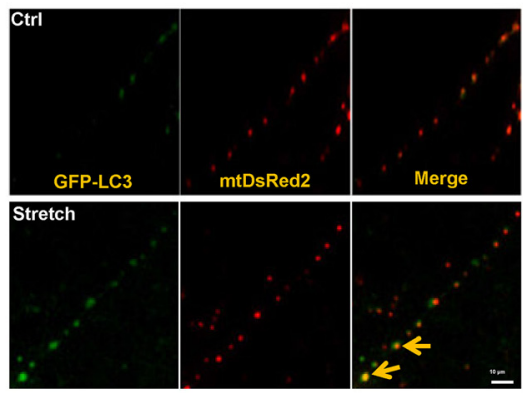

B

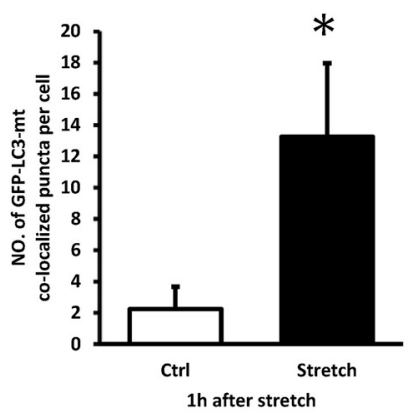

E
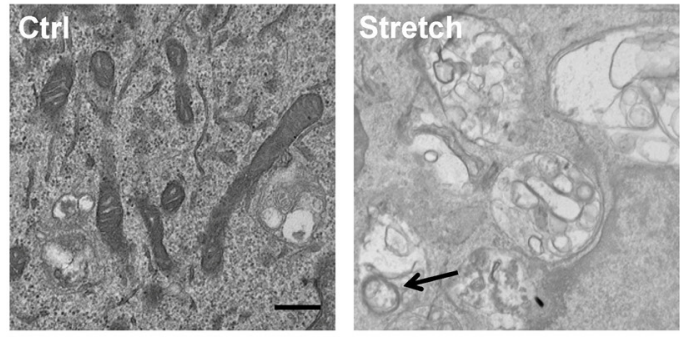

D

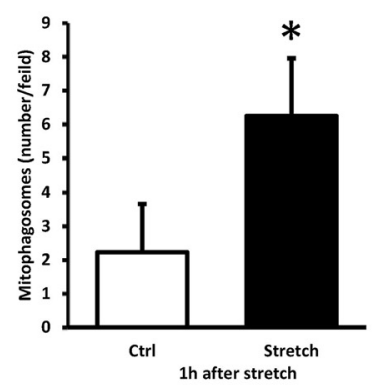

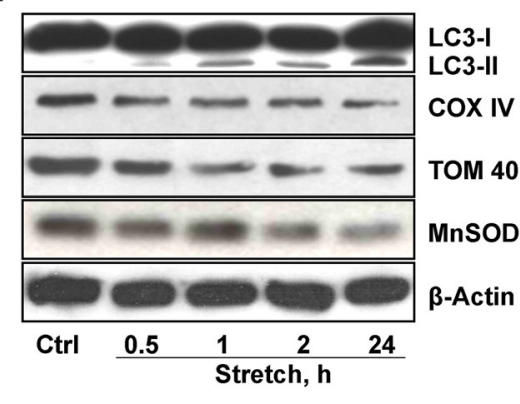

F

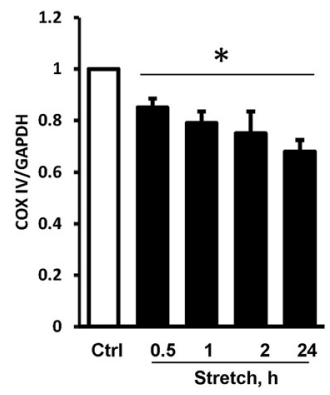

G

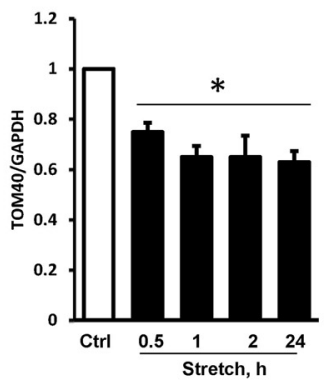

H 
A

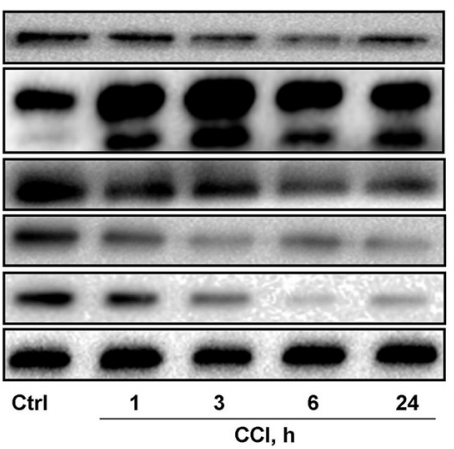

D
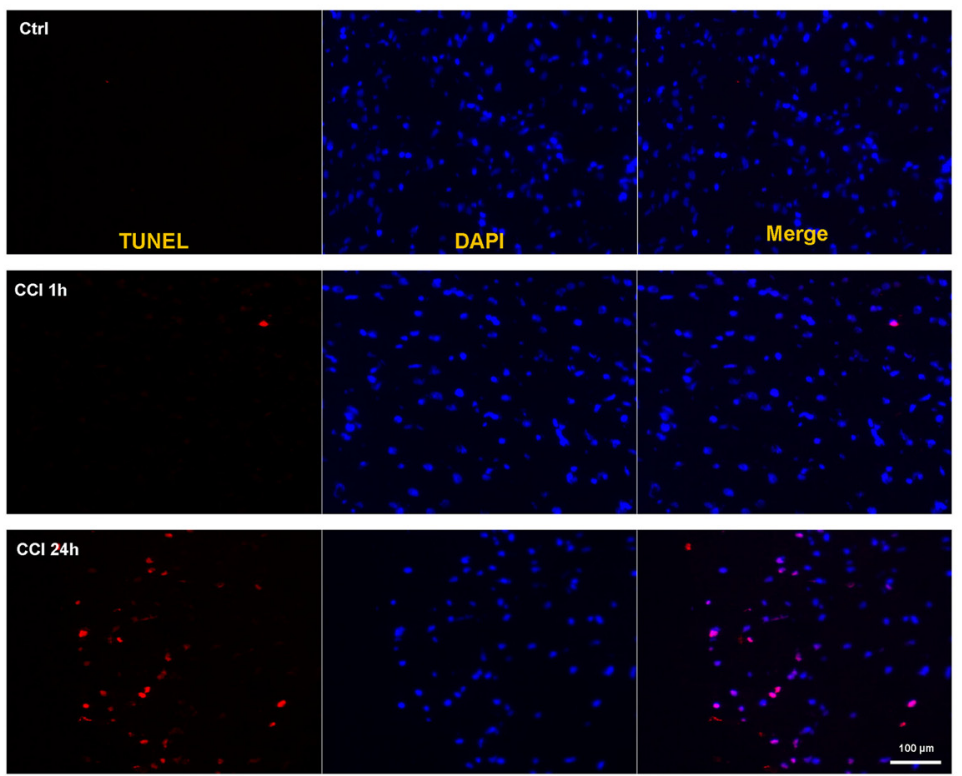

C

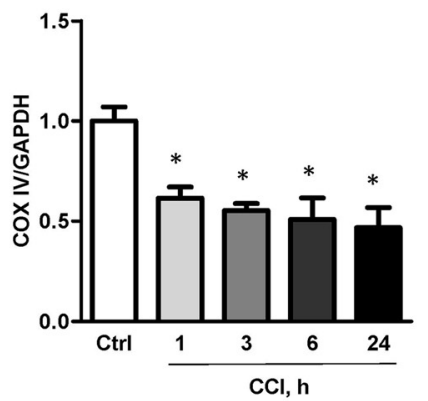

$\mathbf{E}$

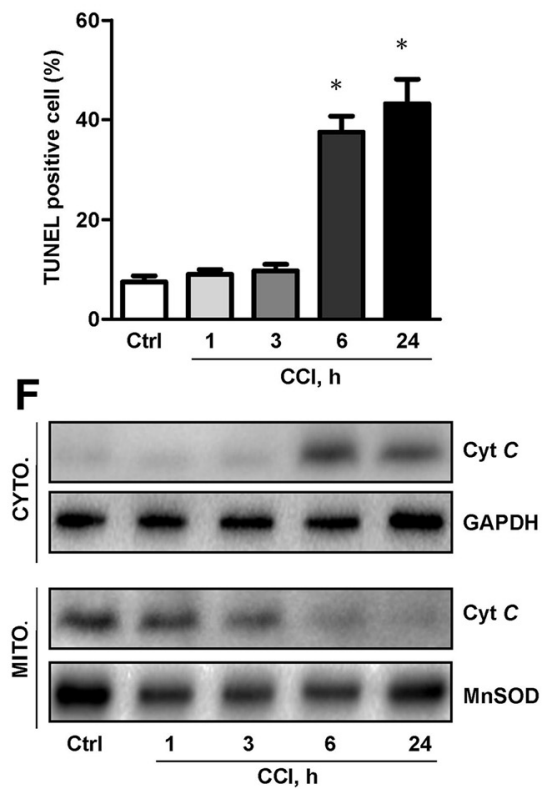

Figure 3. Temporal course of mitophagy and apoptosis after TBI. A-C, Both general autophagy assessed by LC3-Il expression and mitophagy assessed by loss of OMM (TOM 40), IMM (COXIV), and matrix (MnSOD) proteins were induced in the cortex as early as $1 \mathrm{~h}$ after $C\left(\mathrm{Cl}\right.$ and persisted for $24 \mathrm{~h}$ after injury $\left[(\boldsymbol{B}) n=5\right.$; mean $\pm S D ; F_{(4,20)}=16.334$; ${ }^{*} p<0.05$ vs $C$ trl by one-way ANOVA and Tukey's post hoc test; $(\boldsymbol{C})$ mean $\pm S D ; F_{(4,20)}=7.617 ;{ }^{*} p<0.05$ vs Ctrl by one-way ANOVA and Tukey's post hoc test]. $\boldsymbol{D}-\boldsymbol{F}$, Apoptosis assessed by TUNEL staining $(n=5)$ and release of cyt c from mitochondria to cytosol in contusional cortex did not occur until $6 \mathrm{~h}$ after $\mathrm{CII}\left(n=5\right.$; mean $\pm S D ; F_{(4,20)}=23.143 ;{ }^{*} p<0.05$ vs (trl by one-way ANOVA and Tukey's post hoc test). Scale bar, 100 $\mu \mathrm{m}$. CYTO, Cytosolic fraction; MITO, mitochondrial fraction.

formation. Stretch injury enhanced colocalization of GFP-LC3positive puncta with MitoTracker Red, indicating an increased number of mitophagosomes (Fig. 2A,B). Furthermore, injured neurons exhibited double-membrane autophagosomes with encapsulated mitochondria (Fig. 2C,D) and decreased levels of OMM (TOM40), IMM (COXIV), and matrix (MnSOD) proteins (Fig. 2E$H)$. Overall, these data demonstrate the clearance of mitochondria by autophagy after clinical and experimental TBI.

\section{Temporal course of mitophagy after CCI.}

Neuronal apoptosis is a well known contributor to the CCI pathogenesis and our previous work established its association with CL oxidation catalyzed by the cyt $c / C L$ complexes that precedes the release of cyt $c$ into the cytosol (Kagan et al., 2005). Assuming the pro-survival nature of the autophageal elimination of damaged mitochondria, we next studied the time course of mitophagy and apoptosis after CCI. An increase in LC3-II/ GAPDH and decrease in p62/GAPDH were observed as early as $1 \mathrm{~h}$ after CCI versus control, and these changes persisted at $24 \mathrm{~h}$ post-injury (Fig. $3 A, B$ ). The loss of mitochondrial proteins and the colocalization of LC3-II with mitochondria were also apparent as early as $1 \mathrm{~h}$ and persisted until $24 \mathrm{~h}$ after CCI in the injured cortex (Fig. $3 A, C$ ). A similar time course for the selected biomarkers of mitophagy was observed in the CCI injured hippocampus (Fig. $4 A-E$ ). In contrast, TUNEL positivity (Fig. $3 D, E$ ) and cyt $c$ release from the mitochondria (Fig. $3 F$ ) were not observed until $6 \mathrm{~h}$ after CCI. These assessments indicate that mitophagy occurs before the injured cells commit to triggering the death program in response to trauma.

\section{PLS3 deficiency attenuates TBI-induced mitophagy by} inhibiting CL externalization

We have previously shown that the signaling function of CL in mitophagy is realized via its externalization to the surface of mitochondria, where it specifically interacts with the autophagic protein LC3 (Kagan et al., 2005; Chu et al., 2013). Therefore, we next analyzed CL redistribution after TBI, using annexin V-FITC externalization assay to monitor the content of accessible CL on the surface as described previously (Chu et al., 2013; Kagan et al., 2016). Robust externalization of CL on mitochondria was de- 
A

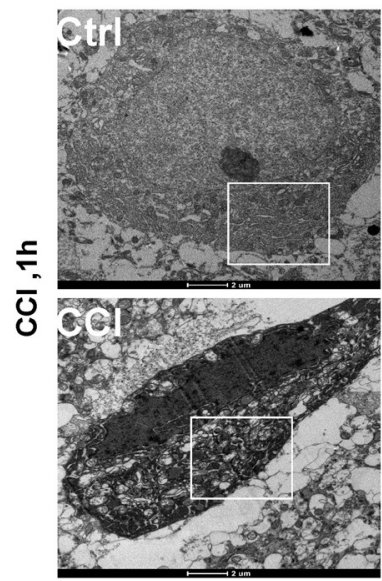

C

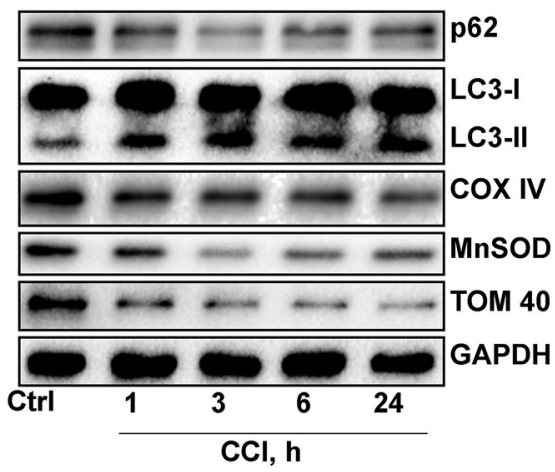

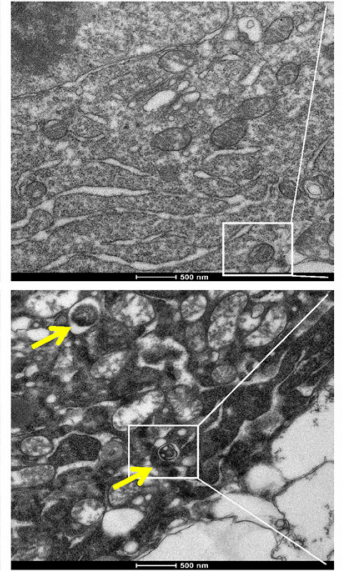

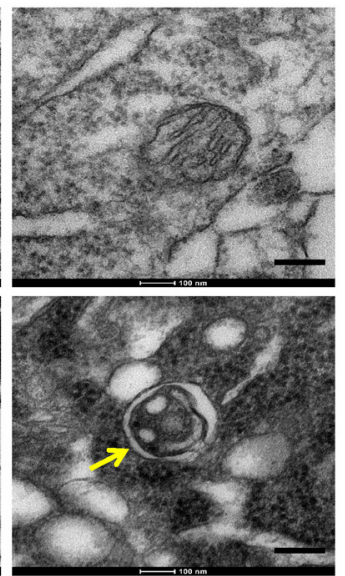

D

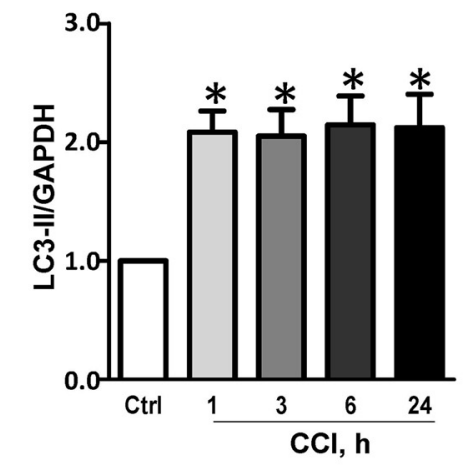

B

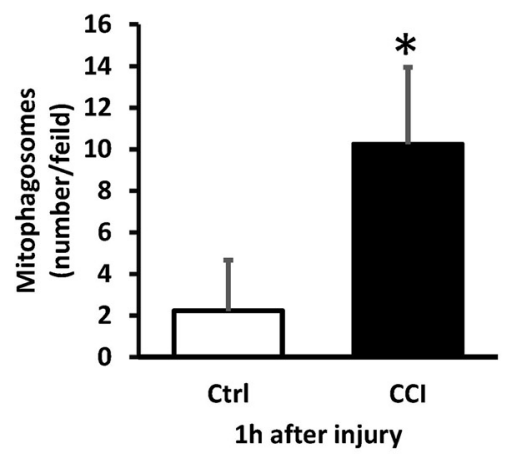

E

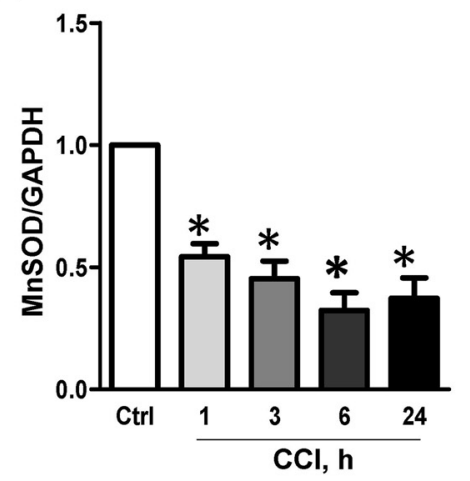

Figure 4. Mitophagy in the hippocampus after TBI. $A$, Representative electron micrographs of injured and sham neurons at $1 \mathrm{~h}$ after injury. A mitophagosome containing partially degraded mitochondria is indicated by the arrow. Scale bar, $0.1 \mu \mathrm{m} . \boldsymbol{B}$, Mitophagosome number was increased after CCl compared with control ( $n=3$; mean $\pm S D ; t=13.761 ; * p<0.05 \mathrm{vs}$ control by $t$ test). $\boldsymbol{C}-\boldsymbol{E}$, The levels of autophagic and mitochondrial markers were assessed by Western blot after TBI $\left[(\boldsymbol{D}) n=3\right.$; mean \pm SEM; $F_{(4,10)}=5.417 ;{ }^{*} p<0.05$ vs control by one-way ANOVA and Tukey's post hoc test; $(\boldsymbol{E}) n=3$; mean $\pm \mathrm{SEM} ; F_{(4,10)}=13.672 ;{ }^{*} p<0.05$ vs control by one-way ANOVA and Tukey's post hoc test].

tected in the human brain after TBI compared with the control group (Fig. 5A).

PLS3 was reported to act as a mitochondrial enzyme responsible for translocating CL from the IMM to the OMM in vitro (Liu et al., 2008) with the accompanying phosphorylation of PLS3 at threonine 21 (Liu et al., 2003a; He et al., 2007). Immunoprecipitation with a PLS3 antibody followed by Western blot with an anti-phosphothreonine antibody showed that PLS3 was phosphorylated after trauma both in vitro and in vivo (Fig. $5 B, G$ ). To determine the role of PLS3 in TBI-induced CL externalization and mitophagy, we continuously administered anti-PLS3 siRNA or nontargeted siRNA intraventricularly for $72 \mathrm{~h}$ to rats before CCI. PLS3 expression was significantly decreased by the targeted siRNA versus nontargeted siRNA in the injured cortex (Fig. 5D). Notably, deficiency of PLS3 markedly suppressed CL externalization induced by trauma both in vitro and in vivo (Fig. 5C,H). Furthermore, PLS3 knockdown prevented CCI-induced loss of mitochondrial proteins without affecting general autophagy markers (Fig. $5 D-F$ ).

\section{Knockdown of CL synthase attenuates mitophagosome} biogenesis after TBI.

To further evaluate the role of $\mathrm{CL}$ in the recognition of injured mitochondria for mitophagy after TBI, we used RNAi against CLS in vitro and in vivo. The RNAi knockdown of CLS resulted in a significantly decreased CLS protein level, but did not affect the expression of COXIV or TOM40 in in vitro and LC3-II in in vivo (Fig. 6). Importantly, we observed that CLS deficiency attenuated mechanical stretch-induced loss of mitochondrial proteins (Fig. 6A-C).

To evaluate the effect of CLS knockdown on TBI-induced mitophagy in vivo, we continuously infused anti-CLS or nontargeted control siRNA into the ventricular system of rats for $72 \mathrm{~h}$ before CCI. CLS expression was significantly decreased (Fig. 6D) by targeted siRNA treatment. Thus achieved CLS knockdown prevented TBI-induced loss of mitochondrial marker proteins, but not general autophagy proteins markers (Fig. 6E,F).

To evaluate mitophagosome formation after TBI, we used GFP-LC3 transgenic rats and assessed the colocalization of GFPLC3 with mitochondria by confocal microscopy. Anti-CLS siRNA administration markedly decreased CCI-induced mitophagosome formation in the injured cortex versus nontargeting siRNA controls (Fig. 6G,H).

\section{Mitophagy is beneficial after TBI by preventing apoptotic death}

Mitochondrial fragmentation is essential in mitophagic processing, in which dynamin-related protein 1 is a key regulator of mitochondrial fission and subsequent elimination by autophagy (Twig et al., 2008; Zhang et al., 2013). We found that mdivi-1, an inhibitor of dynamin-related protein 1 , significantly reversed CCI-induced mitophagy, but did not affect general autophagy (Fig. $7 A-C$ ). 
A

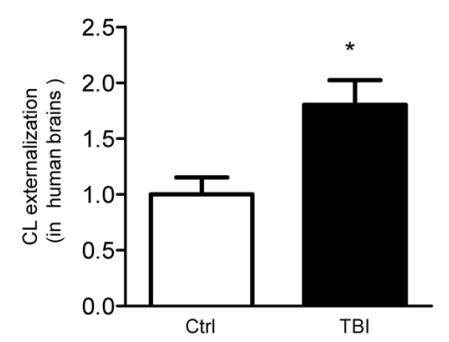

D

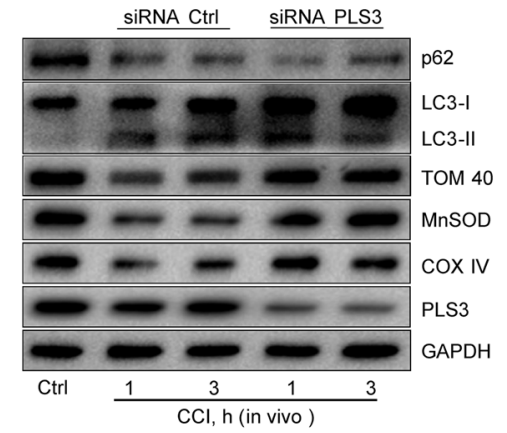

B

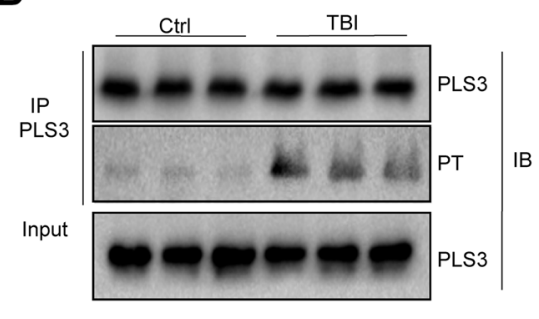

E

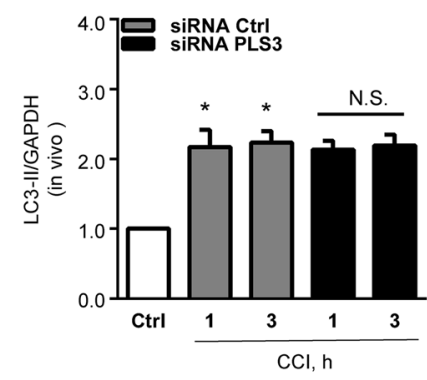

C

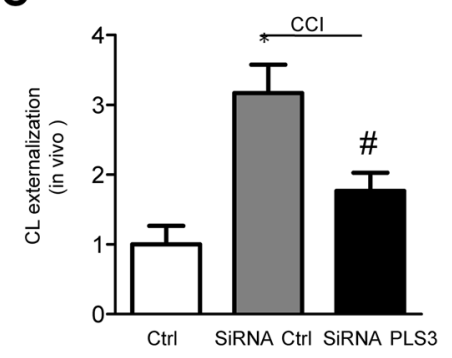

F

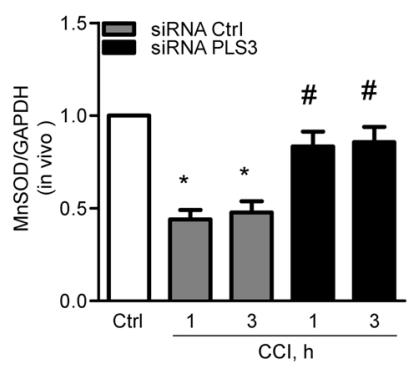

G

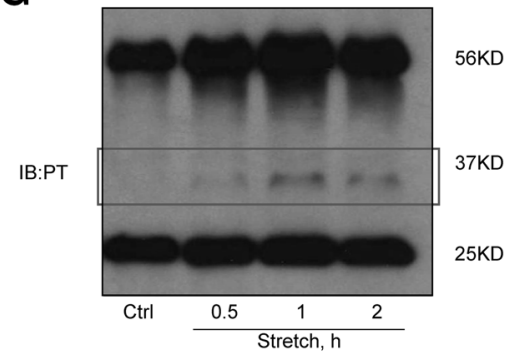

H

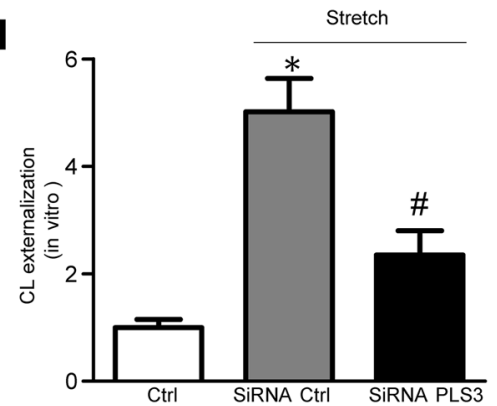

Figure 5. PLS3 knockdown inhibits TBI-induced mitophagy. $\boldsymbol{A}$, Exposure of $\mathrm{CL}$ on mitochondrial surface and phosphorylation of PLS3 (B) are increased in human brains after TBI. (Ctrl $n=3$ and $\mathrm{TBI} n=5$; mean $\pm \mathrm{SD} ; t=2.534 ; p<0.05$ vs Ctrl by $t$ test). C, PLS3 knockdown significantly attenuated C(I-induced CL externalization $\left(n=5 ;\right.$ mean $\pm S D ; F_{(2,12)}=10.530 ;{ }^{*} p<0.05$ vs control and $\# p<0.05$ vs control by one-way ANOVA and Tukey's post hoc test). $\mathbf{D}-\boldsymbol{F}$, CCl elicited increases in LC3-II levels and decreases in mitochondrial protein content, which was reversed by siRNA knockdown of PLS3. Note that PLS3 knockdown had no effect on the expression of the general autophagy marker LC3-II $\left[n=5 ;(\boldsymbol{E})\right.$ mean \pm SEM; $F_{(4,20)}=3.513 ;{ }^{*} p<0.05$ vs $\mathrm{Ctrl}$ and $p>0.05$ $C \mathrm{Cl}+$ siRNA PLS3 vs CCI + siRNA Ctrl by one-way ANOVA and Tukey's post hoc test; $(\boldsymbol{F})$ mean $\pm S E M ; F_{(4,20)}=13.835 ;{ }^{*} p<0.05$ vs Ctrl and \#p $<0.05$ vs $C(\mathrm{Cl}+$ siRNA Ctrl by one-way ANOVA and Tukey's post hoc test]. G, Traumatic stimuli activated PLS3 phosphorylation and $(\boldsymbol{H})$ increased surface exposure of $\mathrm{CL}$ at $3 \mathrm{~h}$ after injury in stretch model $\left(n=5 ;\right.$ mean $\pm S E M ; F_{(2,12)}=23.153 ;{ }^{*} p<$ 0.05 vs Ctrl and \#p $<0.05$ vs (Cl + siRNA Ctrl by one-way ANOVA and Tukey's post hoc test). PT, Phosphothreonine; IP, immunoprecipitation; IB, immunoblotting; PLS3, phospholipid scramblase-3.

Because mitochondrial quality control is essential for neuronal health, we reasoned that impaired mitophagy might trigger or enhance neuronal death after TBI. In line with this notion, treatment of rats with mdivi-1 or 3-MA significantly increased the number of TUNEL-positive cells and markedly upregulated cleaved caspase-3 expression in the injured cortex (Fig. $7 D-F$ ). To further evaluate the effect of mdivi-1, we examined the lesion volume and neurocognitive outcomes. Cortical lesion volume was larger in the mdivi-1-treated rats compared with the rats that received the vehicle (Fig. $7 G$ ). The results of beam balance and MWM tests revealed that the TBI + mdivi-1 group of rats performed markedly worse than the TBI + vehicle group (Fig. $7 H, I)$. To further assess memory retention, we used the novel object recognition (NOR) test. We found that mdivi-1-treated rats spent significantly less time exploring the novel object than the vehicle-treated rats (Fig. $7 \mathrm{~J}$ ) suggesting that inhibition of mitophagy after TBI has a detrimental effect on cognitive function. Motor function before surgery and performance in the visible platform test did not differ between groups. Because both 3-MA and mdivi-1 have off-target effects (Wu et al., 2010; Qian et al.,
2013), we used in vivo PLS3 knock-down protocol to assess the role of CL-mediated mitophagy in the outcome after TBI. The efficacy of PLS3 knockdown was evaluated by Western blot (Fig. $8 B$ ). We used FJC staining, which detects degenerating neurons, to assess lesion evolution in the subacute period ( 1 week after the injury). PLS3 knockdown increased FJC-positivity after CCI compared with nontargeted control siRNA group (Fig. 8A,B). We measured caspase- 3 activity in pericontusional region to evaluate apoptotic markers downstream of mitochondriamediated apoptosis. Greater caspase-3 activity was observed in PLS3 knockdown versus nontargeted control siRNA group after CCI (Fig. 8C). Meanwhile, lesion volume in both cortical and hippocampal tissues was larger PLS3 downregulation compared with the siRNA ctrl (Fig. 8D). After injury, a significant improvement in motor performance was observed on Days 4-5 in the CCI + siRNA Ctrl group compared with the CCI + siRNA PLS3 group (Fig. $8 E$ ). The results of the MWM task revealed that rats in the CCI + siRNA Ctrl group performed better than those in the CCI + siRNA PLS3 group on Days 14-15 (Fig. 8F). PLS3 siRNAtreated rats spent less time with the novel object compared with 
A

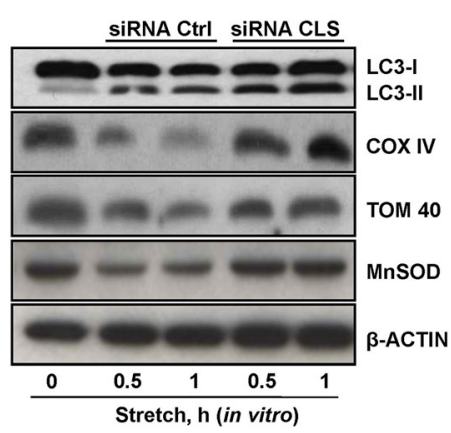

D

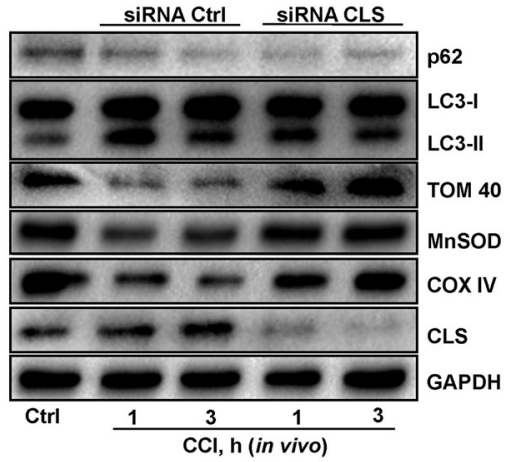

G

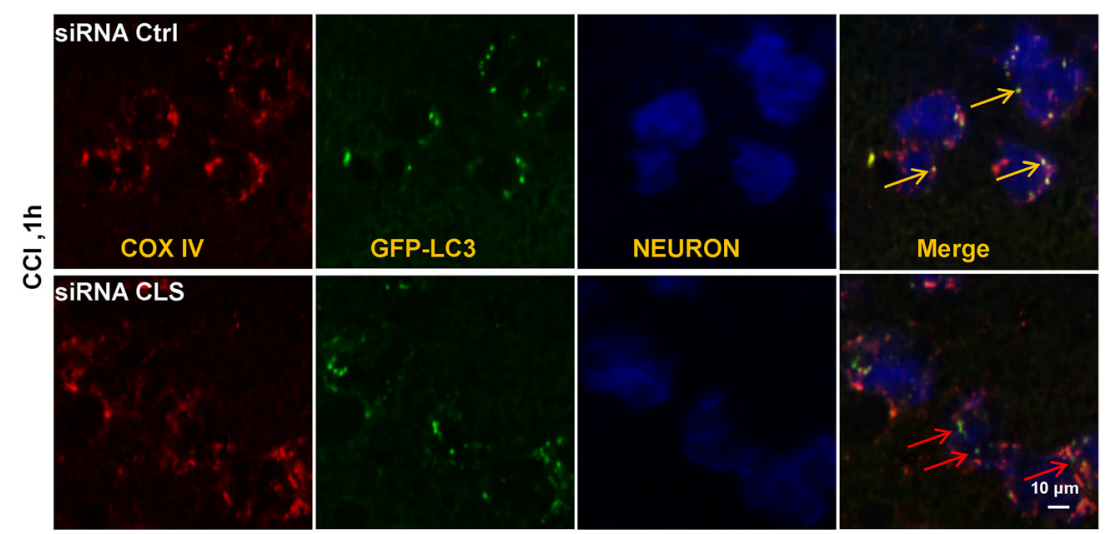

B

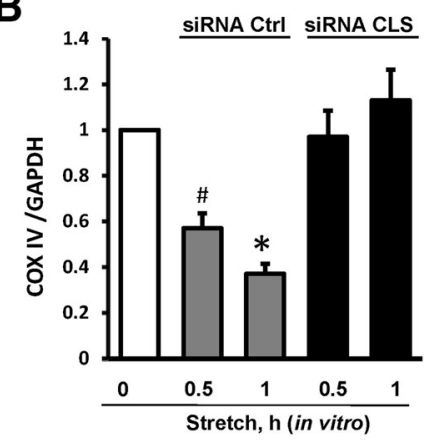

E

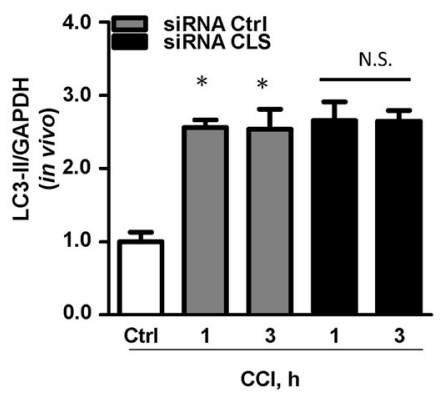

H

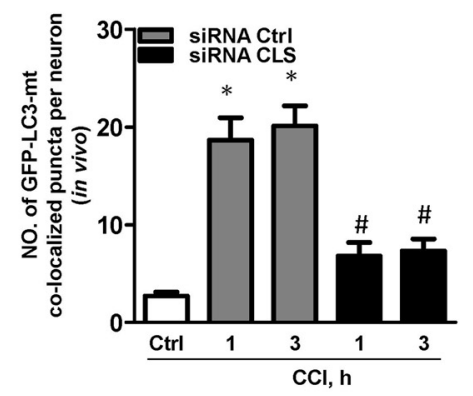

Figure 6. Analysis of $\mathrm{CL}$-mediated mitophagy in response to traumatic injury. $\boldsymbol{A}-\boldsymbol{C}, \mathrm{TB}$-induced decrease of mitochondrial proteins was reversed by siRNA targeting $\mathrm{CLS}$ in in vitro experiment $[(\boldsymbol{B})$ $n=5$; mean $\pm S D ; F_{(4,20)}=13.732 ;{ }^{*} p<0.05$ vs $1 \mathrm{~h}$ with siRNA CLS and $\# p<0.05$ vs $0.5 \mathrm{~h}$ with siRNA CLS by one-way ANOVA and Tukey's posthoc test; $(\boldsymbol{C}) n=5 ;$ mean \pm SEM; $F_{(4,20)}=9.538$; ${ }^{*} p<0.05$ vs $1 \mathrm{~h}$ with siRNA CLS and \#p $<0.05$ vs $0.5 \mathrm{~h}$ with siRNA CLS by one-way ANOVA and Tukey's post hoc test]. $\boldsymbol{D}-\boldsymbol{F}$, Knockdown of CLS attenuated mitophagy after CCI. Both general autophagy assessed by LC3-II expression and mitophagy assessed by loss of OMM (TOM 40) and IMM (COX IV) proteins were induced in cortex at 1 and $3 \mathrm{~h}$ after injury [ $n=3 ;$ ( $\boldsymbol{E}$ ) mean \pm SEM; $F_{(4,10)}=10.679 ;{ }^{*} p<0.05$ vs Ctrl and $p>0.05 C\left(C I+\right.$ siRNA PLS3 vs CCI + siRNA Ctrl by one-way ANOVA and Tukey's post hoc test; $(\boldsymbol{F})$ mean \pm SEM; $F_{(4,10)}=15.139 ;{ }^{*} p<0.05$ vs $C$ trl and \#p $<$ 0.05 vs CCI + siRNA Ctrl by one-way ANOVA and Tukey's post hoc test]. $\mathbf{G}, \boldsymbol{H}$, Mitophagy was assessed by colocalization of GFP-LC3 with mitochondrial protein COX-IV $(n=5)$ in GFP-LC 3 transgenic rats transfected for $72 \mathrm{~h}$ with CLS siRNA or control siRNA intracerebroventricularly. Neuronal nuclei were stained with anti-NeuN antibody in blue $\left(\right.$ mean $\pm S D ; F_{(4,20)}=25.460 ;{ }^{*} p<0.05$ vs control by $t$ test; $\# p<0.05$ vs CCl + siRNA Ctrl by one-way ANOVA and Tukey's post hoc test). Scale bar, $10 \mu \mathrm{m}$. CLS, cardiolipin synthase.

nontargeted siRNA controls (Fig. 8G). These data are compatible with a beneficial role of mitophagy, leading to the clearance of damaged mitochondria and inhibition of downstream apoptosis, after TBI (Fig. 8H).

\section{Discussion}

Primary injury to the brain in the setting of severe TBI in the highly organized and cybernetic machinery of the brain triggers sophisticated repair mechanisms (Park et al., 2008). The complexity of mitochondria as bioenergetic and signaling platforms in neurons makes them particularly vulnerable to mechanical injury that, combined with their high oxidant generating potential, necessitates activation of urgent repair mechanisms. Here we demonstrate, for the first time, that mitophagy is involved in the elimination of damaged organelles as an early TBI response of the injured human and rat brain. This response includes translocation of CL from the IMM to the surface of the OMM where externalized CL mediates targeted LC3-mediated autophagy of damaged mitochondria. Our data targeting PLS and CLS in genetically manipulated cells and animals strongly support the essential role of CL and its externalization mechanisms in the 
A

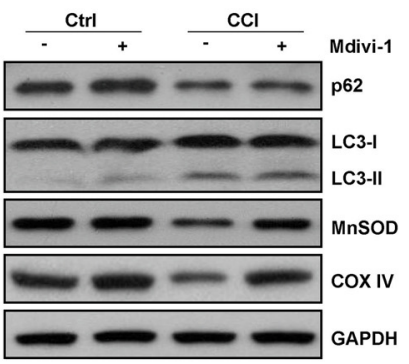

D
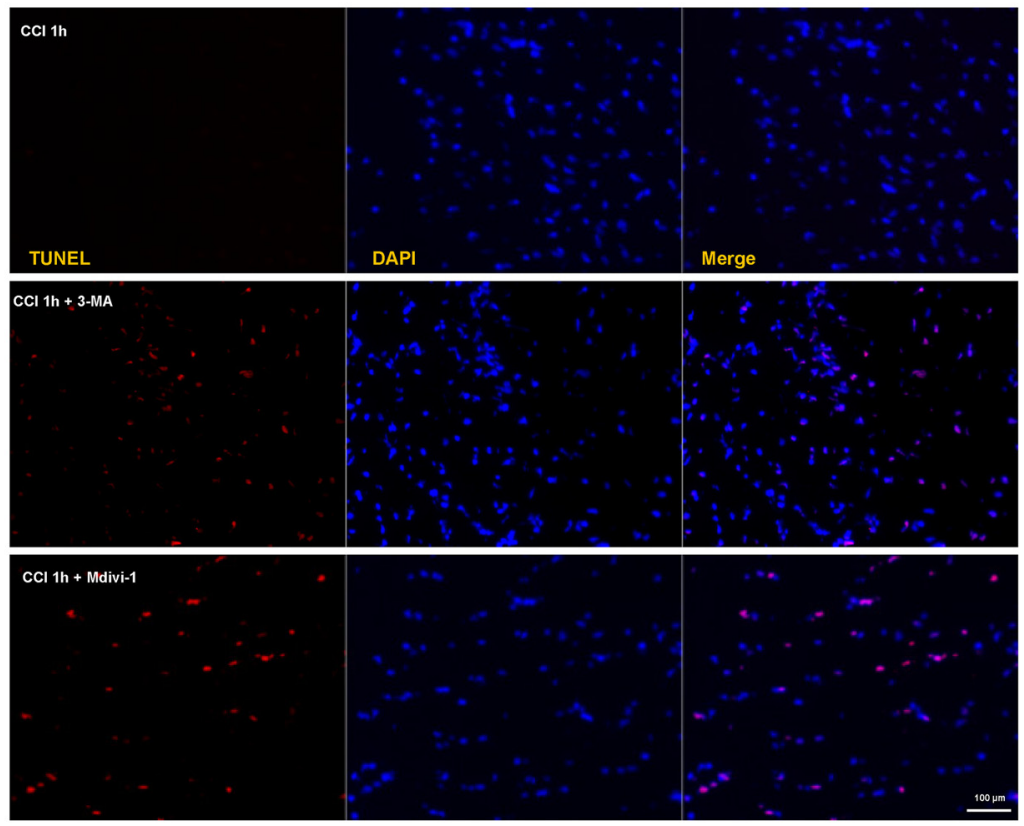

H

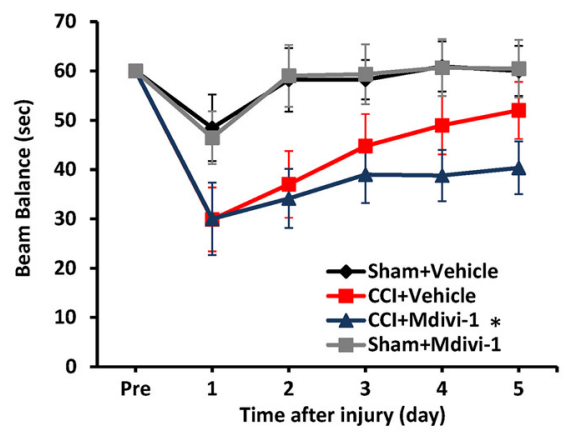

B

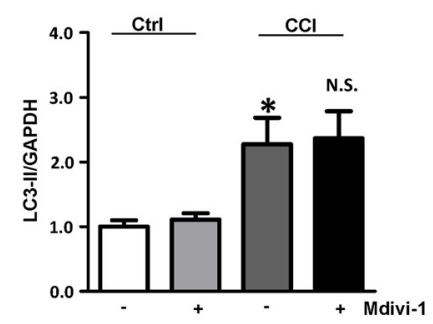

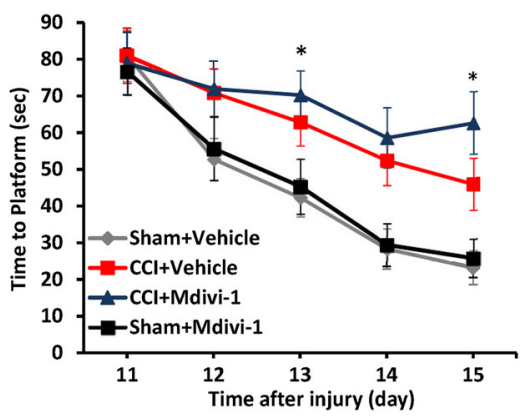

C

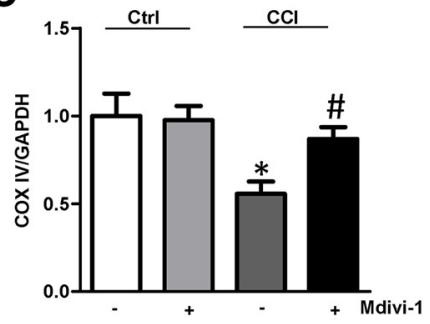

E

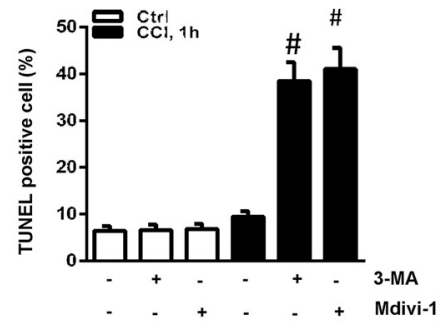

F

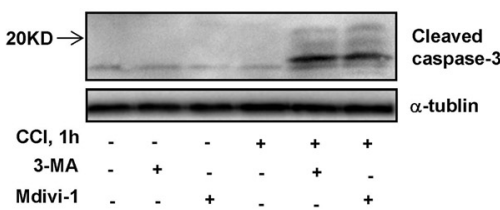

G
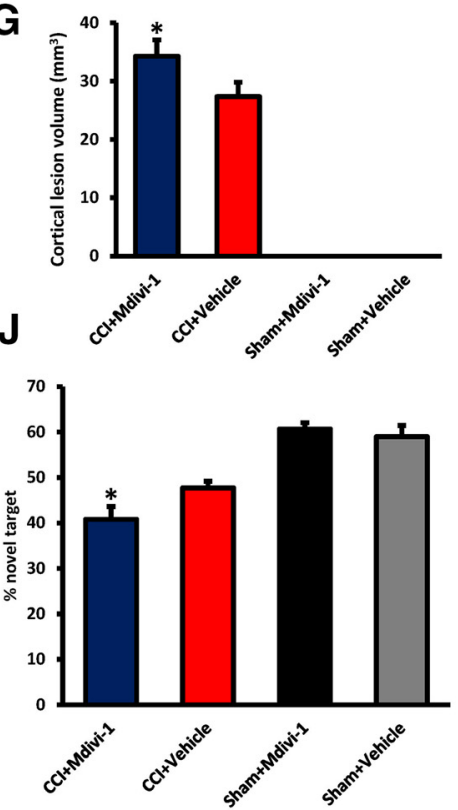

Figure 7. Suppression of mitophagy worsens TBI-induced neuronal injury and behavioral deficits. $A, B$, Mdivi-1 did not affect general autophagosome biogenesis induced by $C(\mathrm{Cl}$ at $1 \mathrm{~h}$ post-injury $\left(n=3 ;\right.$ mean $\pm S D ; F_{(3,8)}=22.742 ;{ }^{*} p<0.05$ vs control and $p>0.05 \mathrm{CCl}$ vs $\mathrm{CCl}+$ Mdivi- 1 by one-way ANOVA and Tukey's post hoc test). $A, C$, The loss of mitochondrial proteins induced by $C \mathrm{Cl}$ was partly reversed by mdivi-1. GAPDH was used as the loading control ( $n=3$; mean $\pm S D ; F_{(3,8)}=12.583 ;{ }^{*} p<0.05$ vs control and \# $<0.05$ vs $(C I$ by one-way ANOVA and Tukey's post hoc test). $\boldsymbol{D}, \boldsymbol{E}$, Apoptotic cell death was assessed by TUNEL staining from five random fields in the injured cortex $\left(n=5 ;\right.$ mean $\pm S D ; F_{(5,24)}=40.362 ;{ }^{\#} p<0.05$ vs $C(\mathrm{Cl}, 1 \mathrm{~h}$ by $t$ test). Scale bar, $100 \mu \mathrm{m}$. $\boldsymbol{F}$, 3-MA or mdivi- 1 increased the level of cleaved-caspase- 3 after CCI. G, Analysis of cortical lesion volume $7 \mathrm{~d}$ after TBl in Sprague-Dawley rats $\left(n=5 ;\right.$ mean $\pm S D ; F_{(2,15)}=4.163 ;{ }^{*} p<0.05$ vs $C \mathrm{Cl}+\mathrm{V}$ by one-way ANOVA and Tukey's post hoc test). $\boldsymbol{H}$, Time spent (seconds) on the balance beam apparatus before and after CCl or sham $\left(n=8-12 ;\right.$ mean $\pm S D ; F_{(5,300)}=54.362 ;{ }^{*} p<0.05 \mathrm{vs}(C l+V$ ehicle by one-way ANOVA and Tukey's post hoc test). I, Latency (seconds) till rats locate a hidden (submerged) and visible platform on post-TBI Days $11-15\left(n=8-12 ;\right.$ mean $\pm S D ; F_{(5,300)}=72.128 ;{ }^{*} p<0.05$ vs $\mathrm{CCl}+$ Vehicle by one-way ANOVA and Tukey's post hoc test).J, NOR task performance $19 \mathrm{~d}$ after sham or CCl injury ( $n=8-12$ per group; mean \pm SEM; $F_{(5,36)}=9.714 ;{ }^{*} p<0.05$ vs $C \mathrm{Cl}+$ Vehicle by one-way ANOVA and Tukey's post hoc test). V, Vehicle.

endogenous reparative plasticity of injured brain cells. We further show that successful execution and completion of mitophagy is beneficial in the context of preservation of cognitive functions after TBI thus suggesting that mechanisms and path- ways of CL externalization may represent new targets for drug discovery.

Mitophagy as a regulator and precursor of apoptosis has previously been documented in TBI and other CNS disorders (Tang 
A
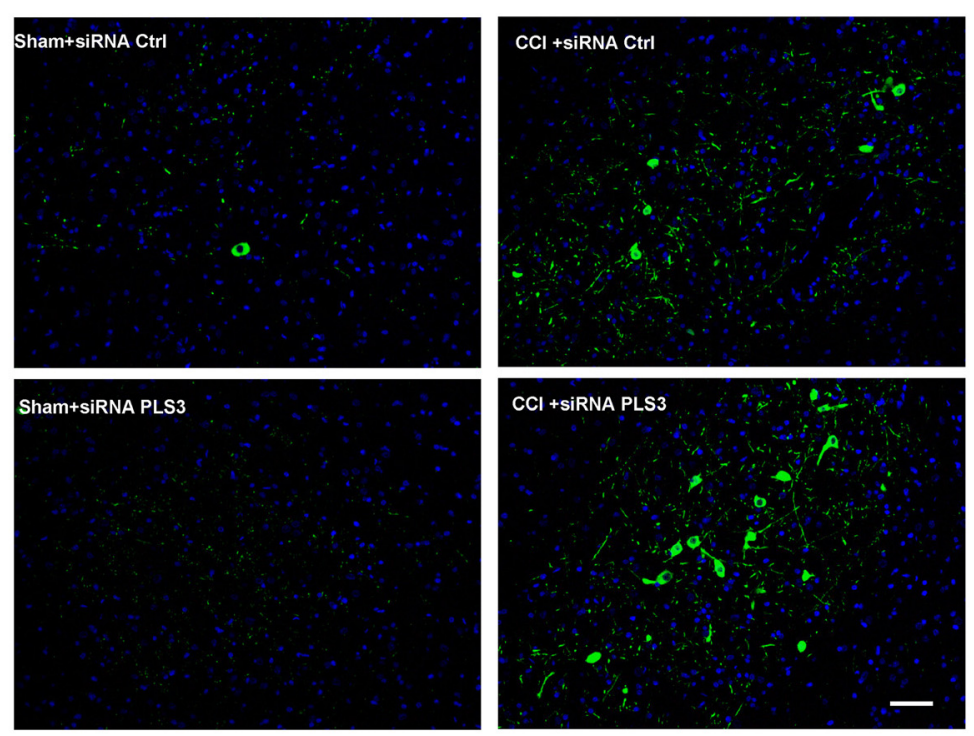

D
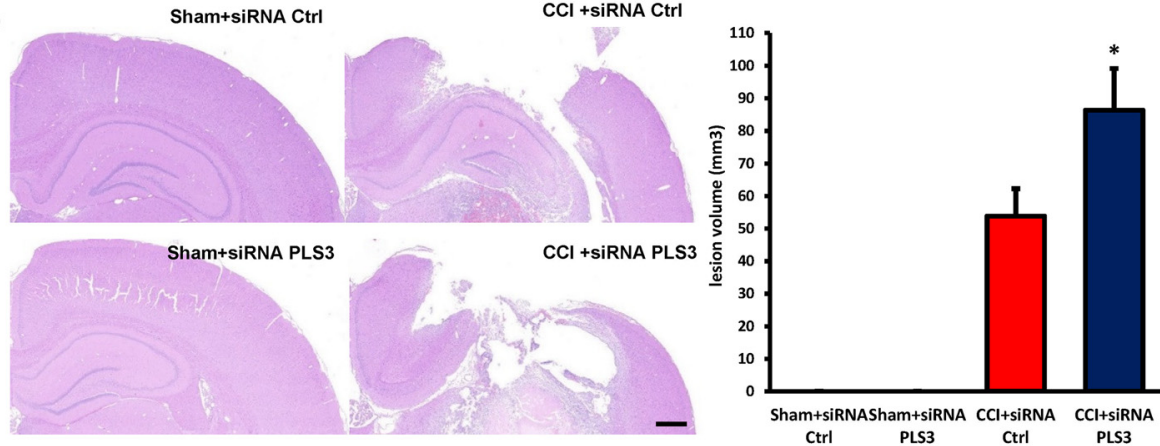

F

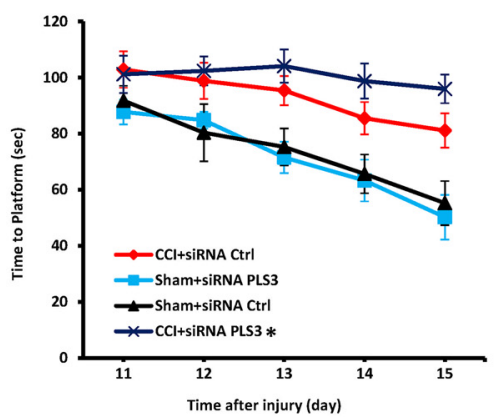

G

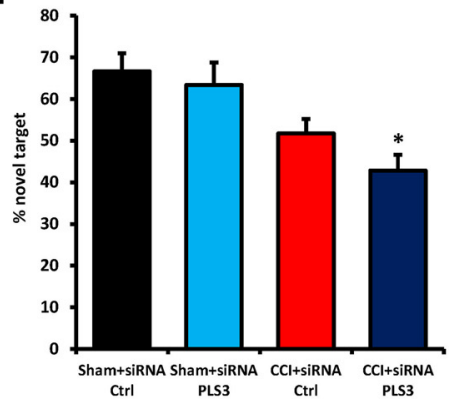

B

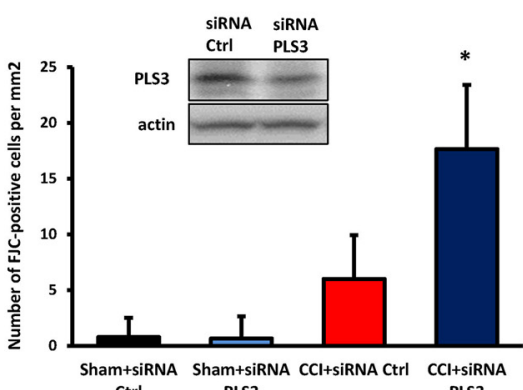

C

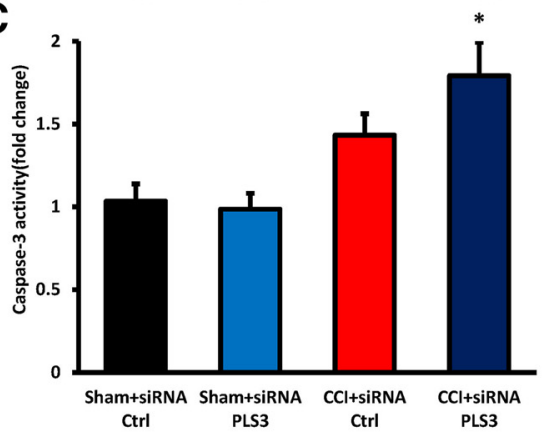

$\mathbf{E}$

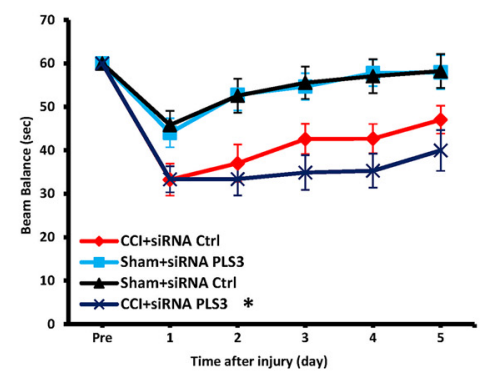

H

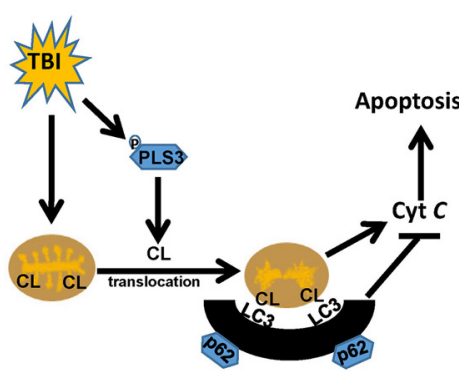

Figure 8. PLS3 knockdown exacerbates neuronal injury and behavioral deficits after TBI. $A$, Assessment of neurodegeneration by FJC staining. Neurodegeneration observed in the injured cortex 1 week after CCI was exacerbated by PLS3 downregulation. Scale bar, $100 \mu \mathrm{m}$. B, Quantification of FJC staining showed significantly higher neurodegeneration in the CCI + siRNA PLS3 group ( $n=$ 5 ; mean $\pm S D ; F_{(3,16)}=32.461 ;{ }^{*} p<0.05$ vs $C(\mathrm{Cl}+$ siRNA (trl by one-way ANOVA and Tukey's post hoc test). Rats were transfected with control or PLS3 siRNA $72 \mathrm{~h}$ before CCl and measurements were obtained $24 \mathrm{~h}$ after injury. Insert: The efficacy of PLS3 knockdown evaluated by Western blot, $n=5 /$ group. C, Caspase-3 activity of pericontusional cortex $\left(n=5 ;\right.$ means $\pm S D ; F_{(3,16)}=17.227$ ${ }^{*} p<0.05$ vs $\mathrm{CCl}+$ siRNA Ctrl by one-way ANOVA and Tukey's post hoc test). $D$, Analysis of lesion volume (both cortical and hippocampal areas using H\&E staining) $7 \mathrm{~d}$ after $C \mathrm{Cl}(n=5 ;$ mean \pm $S D ; F_{(3,16)}=10.325 ;{ }^{*} p<0.05$ vs $(C l+V$ by one-way ANOVA and Tukey's post hoc test). Scale bar, $500 \mu \mathrm{m}$. $\boldsymbol{E}$, Time spent (in seconds) on the balance beam apparatus before and after $C(\mathrm{Cl}$ or sham $\operatorname{injury}\left(n=8-12 ;\right.$ mean $\pm S D ; F_{(3,200)}=72.671 ;{ }^{*} p<0.05$ vs CCI + siRNA Ctrl by one-way ANOVA and Tukey's post hoc test). $F$, Latency (in seconds) till rats locate a hidden (submerged) platform on post-TBI Days $11-15\left(n=8-12 ; F_{(3,200)}=14.271 ;{ }^{*} p<0.05\right.$ vs CCI + siRNA Ctrl by one-way ANOVA and Tukey's posthoctest). G, NOR task performance $19 \mathrm{~d}$ after sham or CCl injury $(n=8-12$; mean \pm SEM; $F_{(3,36)}=45.461 ;{ }^{*} p<0.05$ vs $C(\mathrm{C} 9+$ siRNA (trl by one-way ANOVA and Tukey's post hoc test). $\boldsymbol{H}$, Schematic diagram summarizing the proposed role of $\mathrm{CL}$ in mitophagy after TBI. FJC, Fluoro jade-C.

et al., 2016; Liu et al., 2017b; Wang et al., 2017; Wu et al., 2017). A number of studies have reported presence of abnormal mitochondria early after acute injury, possibly associated with energy depletion and/or disturbed $\mathrm{Ca}^{2+}$ homeostasis (Cheng et al., 2012). Within this context autophagy of mitochondria might play an important positive homeostatic role of in endogenous metabolic rebalancing in acute injury, especially in TBI (Smith et al., 2011). In line with this, the induction of autophagy using rapamycin inhibited an important mitochondrial cell death pathway with decreased cyt $c$ release and caspase 3 activation (Tang et al., 2016; Wang et al., 2017; Wu et al., 2017), while inhibition of mitophagy by mdivi-1-induced cyt $c$ release and caspase 3 activa- 
tion (Zuo et al., 2014). In addition to TBI, dramatically increased mitophagy was observed within hours after reperfusion in both middle cerebral artery occlusion and oxygen and glucose deprivation experiments (Zhang et al., 2013).

Both beneficial and detrimental effects of autophagy or mitophagy have been reported after TBI (Galluzzi et al., 2016; Feng et al., 2017; Gao et al., 2017; Huang et al., 2017; Liu et al., 2017a). To better understand mitophagy and cell death after TBI, we performed a time course assessment of mitochondrial protein content and TUNEL immunofluorescence. We found that after an early period of mitophagy ( $1 \mathrm{~h}, 3 \mathrm{~h}$ ), cells underwent significant cell death with cyt $c$ release from 6 to $24 \mathrm{~h}$. This early onset of mitophagy may reflect the attempts of cells to eliminate the injured mitochondria to circumvent the necessity to engage a more drastic cell response, apoptosis. To experimentally test this idea, we used 3-MA and mdivi-1 as autophagy and mitophagy inhibitors respectively, as well as PLS3 siRNA to specifically block mitophagy in a rat CCI model. Mdivi-1 is a mitochondrial fission inhibitor that blocks mitophagy via decreased fragmentation of mitochondria (Smith and Gallo, 2017). In line with this, Fischer et al. (2016) showed that mdivi-1 blocked CCI-induced decrease in mitochondrial length and mito-fragmentation in the hippocampus (Rosdah et al., 2016). They observed that administration of Mdivi-1 starting 30 min post-injury, and subsequently at 24 and 48 h post-injury improved NOR performance after CCI. These results differ from our findings. One possible explanation for this difference could be the timing of Mdivi-1 administration; post-injury versus pre-injury. Preau et al. (2016) showed that the mitochondrial fragmentation is essential in mitophagic processing. However, other studies indicate that mitochondrial fission is not required for mitophagy (Yamashita et al., 2016; Burman et al., 2017). It is possible that the beneficial versus detrimental effects of mitophagy after TBI depend on timing after injury. 3-MA, an autophagy inhibitor, has been also tested as a response modifier after TBI (Luo et al., 2011) with conflicting results (Wang et al., 2012; Jin et al., 2016). However, the harmful effects of long-term autophagy and continued activation of LC3 cannot be completely excluded by our study focused on the early time points, from 1 to $24 \mathrm{~h}$, after TBI (Kroemer and Levine, 2008; Liu and Levine, 2015). This is an important unanswered question given that Sarkar et al. (2014) demonstrated the persistence of autophagy over the period of 1 week after TBI. We also found a transient motor deficit affecting their beam balance performance on Day 1 after surgery in sham group. These deficits resolve by Day 2 after surgery. Sham operated rats undergo craniotomy and anesthesia and it is possible that these procedures influence beam balance performance on Day 1 after surgery. Previous studies have shown that craniotomy alone can cause mild transient neurological dysfunction. Nevertheless it is thought that the effects of surgery are best controlled by including surgical sham control groups (Cole et al., 2011; Osier and Dixon, 2016).

Our previous studies showed that mitochondria are important determinants of the ultimate fate of cells after TBI (Bayir et al., 2007; Ji et al., 2012a). We demonstrated that cyt $c$ release from mitochondria triggered by stretch injury in primary neurons was causatively linked with selective oxidation of CL (Kagan et al., 2009). Although translocation of CL from the IMM to the OMM is required by both apoptosis and mitophagy, its oxidation by the cyt $c / \mathrm{CL}$ complex in the presence of hydrogen peroxide is essential for mitochondrial apoptosis (Kagan et al., 2005). Appearance of CL on the surface of damaged mitochondria acts as an eat me signal for the autophageal machinery via recognition of externalized CL by LC3 (Chu et al., 2013, 2014). Availability of CL likely represents a key factor for its externalization. In line with this possibility, our data showed that knockdown of CL synthase, the enzyme responsible for its de novo synthesis, reduced mitophagy. Importantly, quantification of LC3/GAPDH showed no significant difference between groups treated with either siRNA-control or siRNA-CL, suggesting that overall autophagy was not affected by CLS deletion.

Another protein that plays an important role in the translocation of CL from the inner to the outer mitochondrial membrane during mitophagy is PLS3 (Liu et al., 2003b). It has been shown that lipid transfer activity of PLS3 is increased by phosphorylation of its Thr 21 by protein kinase C (PKC)- $\delta$ (He et al., 2007). Previous studies have shown enhanced expression of PLS3 in hippocampal mitochondria and colocalization of PLS3 with PKC- $\delta$ after cerebral ischemia reperfusion (Kowalczyk et al., 2009). Our result in the CCI model also show increased phosphorylation of PLS3 accompanied by markedly elevated levels of CL in OMM fractions isolated from injured brain. As overexpression of PLS3 has been shown to enhance CL translocation and increase sensitivity to apoptosis (Liu et al., 2003b), we reasoned that PLS3 also played a role in mitophagy after TBI. Indeed, we demonstrated that anti-RNAi injection in vivo resulted in knockdown of PLS3 and downregulated mitophagy in the CCI model. Acting as a multifactorial regulator of both the number and function of mitochondria, CL can also interact with the autophagy protein beclin 1, the immunity-related GTPase IRGM, and NLRP3 (Geisler et al., 2010; Sun et al., 2012; Iyer et al., 2013). Overall, our results show that CL signaling is essential for the quality control of mitochondria via the mitophageal elimination of TBI damaged organelles.

There are several limitations to our study. First being focused on the involvement of CL in the mitophageal elimination of damaged mitochondria, this study did not explore the possible contribution of PINK1/Parkin (Mukhida et al., 2005; Zhang et al., 2013; Wang et al., 2017) to TBI-induced mitophagy in the brain. Second, we used a developmental TBI model in juvenile rats whereas the clinical samples were obtained from adult patients. Related to clinical samples, epilepsy patients were used as controls and were thus not true normal naive controls, but represented the best available source of control samples given that resection of brain tissue from normal healthy controls is unethical. Furthermore, clinical TBI tissues were mostly frontal, whereas all control tissues were temporal. In addition, the mean age of the controls was younger than in the TBI patients. Nevertheless, these are extremely valuable human samples that represent the best available control tissue for our studies.

In summary, we report that activation of CL-dependent mitophagy is an essential component of the responses elicited by clinical and experimental TBI. Suppression of TBI-induced mitophagy worsens the overall outcome likely via increased apoptosis. Our findings are important for better understanding of the mechanisms of secondary injury after TBI and could guide the discovery of new small molecule modulators of mitophagy that could serve as potential novel therapeutics for TBI and other acute CNS insults.

\section{References}

Bayir H, Tyurin VA, Tyurina YY, Viner R, Ritov V, Amoscato AA, Zhao Q, Zhang XJ, Janesko-Feldman KL, Alexander H, Basova LV, Clark RS, Kochanek PM, Kagan VE (2007) Selective early cardiolipin peroxidation after traumatic brain injury: an oxidative lipidomics analysis. Ann Neurol 62:154-169.

Burman JL, Pickles S, Wang C, Sekine S, Vargas JNS, Zhang Z, Youle AM, Nezich CL, Wu X, Hammer JA, Youle RJ (2017) Mitochondrial fission 
facilitates the selective mitophagy of protein aggregates. J Cell Biol 216:3231-3247.

Cavallucci V, Bisicchia E, Cencioni MT, Ferri A, Latini L, Nobili A, Biamonte F, Nazio F, Fanelli F, Moreno S, Molinari M, Viscomi MT, D’Amelio M (2014) Acute focal brain damage alters mitochondrial dynamics and autophagy in axotomized neurons. Cell Death Dis 5:e1545.

Cheng G, Kong RH, Zhang LM, Zhang JN (2012) Mitochondria in traumatic brain injury and mitochondrial-targeted multipotential therapeutic strategies. Br J Pharmacol 167:699-719.

Chu CT, Ji J, Dagda RK, Jiang JF, Tyurina YY, Kapralov AA, Tyurin VA, Yanamala N, Shrivastava IH, Mohammadyani D, Wang KZQ, Zhu J, Klein-Seetharaman J, Balasubramanian K, Amoscato AA, Borisenko G, Huang Z, Gusdon AM, Cheikhi A, Steer EK, et al. (2013) Cardiolipin externalization to the outer mitochondrial membrane acts as an elimination signal for mitophagy in neuronal cells. Nat Cell Biol 15:1197-1205.

Chu CT, Bayir H, Kagan VE (2014) LC3 binds externalized cardiolipin on injured mitochondria to signal mitophagy in neurons: implications for Parkinson disease. Autophagy 10:376-378.

Clark RS, Bayir H, Chu CT, Alber SM, Kochanek PM, Watkins SC (2008) Autophagy is increased in mice after traumatic brain injury and is detectable in human brain after trauma and critical illness. Autophagy 4:88-90.

Cole JT, Yarnell A, Kean WS, Gold E, Lewis B, Ren M, McMullen DC, Jacobowitz DM, Pollard HB, O'Neill JT, Grunberg NE, Dalgard CL, Frank JA, Watson WD (2011) Craniotomy: true sham for traumatic brain injury, or a sham of a sham? J Neurotrauma 28:359-369.

Feng Y, Cui C, Liu X, Wu Q, Hu F, Zhang H, Ma Z, Wang L (2017) Protective role of apocynin via suppression of neuronal autophagy and TLR4/ $\mathrm{NF}-\kappa \mathrm{B}$ signaling pathway in a rat model of traumatic brain injury. Neurochem Res 42:3296-3309.

Fischer TD, Hylin MJ, Zhao J, Moore AN, Waxham MN, Dash PK (2016) Altered mitochondrial dynamics and TBI pathophysiology. Front Syst Neurosci 10:29.

Galluzzi L, Bravo-San Pedro JM, Blomgren K, Kroemer G (2016) Autophagy in acute brain injury. Nat Rev Neurosci 17:467-484.

Gao Y, Zhuang Z, Gao S, Li X, Zhang Z, Ye Z, Li L, Tang C, Zhou M, Han X, Li J (2017) Tetrahydrocurcumin reduces oxidative stress-induced apoptosis via the mitochondrial apoptotic pathway by modulating autophagy in rats after traumatic brain injury. Am J Transl Res 9:887-899.

Geisler S, Holmström KM, Skujat D, Fiesel FC, Rothfuss OC, Kahle PJ, Springer W (2010) PINK1/Parkin-mediated mitophagy is dependent on VDAC1 and p62/SQSTM1. Nat Cell Biol 12:119-131.

He Y, Liu J, Grossman D, Durrant D, Sweatman T, Lothstein L, Epand RF, Epand RM, Lee RM (2007) Phosphorylation of mitochondrial phospholipid scramblase 3 by protein kinase C- $\delta$ induces its activation and facilitates mitochondrial targeting of tBid. J Cell Biochem 101:12101221.

Huang CY, Lee YC, Li PC, Liliang PC, Lu K, Wang KW, Chang LC, Shiu LY, Chen MF, Sun YT, Wang HK (2017) TDP-43 proteolysis is associated with astrocyte reactivity after traumatic brain injury in rodents. J Neuroimmunol 313:61-68.

Iyer SS, He Q, Janczy JR, Elliott EI, Zhong Z, Olivier AK, Sadler JJ, KnepperAdrian V, Han R, Qiao L, Eisenbarth SC, Nauseef WM, Cassel SL, Sutterwala FS (2013) Mitochondrial cardiolipin is required for Nlrp3 inflammasome activation. Immunity 39:311-323.

Ji J, Tyurina YY, Tang M, Feng W, Stolz DB, Clark RS, Meaney DF, Kochanek PM, Kagan VE, Bayir H (2012a) Mitochondrial injury after mechanical stretch of cortical neurons in vitro: biomarkers of apoptosis and selective peroxidation of anionic phospholipids. J Neurotrauma 29:776-788.

Ji J, Kline AE, Amoscato A, Samhan-Arias AK, Sparvero LJ, Tyurin VA, Tyurina YY, Fink B, Manole MD, Puccio AM, Okonkwo DO, Cheng JP, Alexander H, Clark RS, Kochanek PM, Wipf P, Kagan VE, Bayir H (2012b) Lipidomics identifies cardiolipin oxidation as a mitochondrial target for redox therapy of brain injury. Nat Neurosci 15:1407-1413.

Jin Y, Lei J, Lin Y, Gao GY, Jiang JY (2016) Autophagy inhibitor 3-MA weakens neuroprotective effects of posttraumatic brain injury moderate hypothermia. World Neurosurg 88:433-446.

Kagan VE, Jiang J, Huang Z, Tyurina YY, Desbourdes C, Cottet-Rousselle C, Dar HH, Verma M, Tyurin VA, Kapralov AA, Cheikhi A, Mao G, Stolz D, St Croix CM, Watkins S, Shen Z, Li Y, Greenberg ML, TokarskaSchlattner M, Boissan M, et al. (2016) NDPK-D (NM23-H4)-mediated externalization of cardiolipin enables elimination of depolarized mitochondria by mitophagy. Cell Death Differ 23:1140-1151.
Kagan VE, Tyurin VA, Jiang J, Tyurina YY, Ritov VB, Amoscato AA, Osipov AN, Belikova NA, Kapralov AA, Kini V, Vlasova II, Zhao Q, Zou M, Di P, Svistunenko DA, Kurnikov IV, Borisenko GG (2005) Cytochrome c acts as a cardiolipin oxygenase required for release of proapoptotic factors. Nat Chem Biol 1:223-232.

Kagan VE, Bayir HA, Belikova NA, Kapralov O, Tyurina YY, Tyurin VA, Jiang J, Stoyanovsky DA, Wipf P, Kochanek PM, Greenberger JS, Pitt B, Shvedova AA, Borisenko G (2009) Cytochrome c/cardiolipin relations in mitochondria: a kiss of death. Free Radic Biol Med 46:1439-1453.

Kilbaugh TJ, Karlsson M, Byro M, Bebee A, Ralston J, Sullivan S, Duhaime AC, Hansson MJ, Elmér E, Margulies SS (2015) Mitochondrial bioenergetic alterations after focal traumatic brain injury in the immature brain. Exp Neurol 271:136-144.

Klionsky DJ, Abdelmohsen K, Abe A, Abedin MJ, Abeliovich H, Acevedo Arozena A, Adachi H, Adams CM, Adams PD, Adeli K, Adhihetty PJ, Adler SG, Agam G, Agarwal R, Aghi MK, Agnello M, Agostinis P, Aguilar PV, Aguirre-Ghiso J, Airoldi EM, et al. (2016) Guidelines for the use and interpretation of assays for monitoring autophagy (3rd edition). Autophagy 12:1-222.

Kowalczyk JE, Beresewicz M, Gajkowska B, Zabłocka B (2009) Association of protein kinase $\mathrm{C}$ delta and phospholipid scramblase 3 in hippocampal mitochondria correlates with neuronal vulnerability to brain ischemia. Neurochem Int 55:157-163.

Kroemer G, Levine B (2008) Autophagic cell death: the story of a misnomer. Nat Rev Mol Cell Biol 9:1004-1010.

Lai Y, Hickey RW, Chen Y, Bayir H, Sullivan ML, Chu CT, Kochanek PM, Dixon CE, Jenkins LW, Graham SH, Watkins SC, Clark RS (2008) Autophagy is increased after traumatic brain injury in mice and is partially inhibited by the antioxidant gamma-glutamylcysteinyl ethyl ester. J Cereb Blood Flow Metab 28:540-550.

Liu HD, Li W, Chen ZR, Zhou ML, Zhuang Z, Zhang DD, Zhu L, Hang CH (2013) Increased expression of ferritin in cerebral cortex after human traumatic brain injury. Neurol Sci 34:1173-1180.

Liu J, Chen J, Dai Q, Lee RM (2003a) Phospholipid scramblase 3 is the mitochondrial target of protein kinase $\mathrm{C}$ delta-induced apoptosis. Cancer Res 63:1153-1156.

Liu J, Dai Q, Chen J, Durrant D, Freeman A, Liu T, Grossman D, Lee RM (2003b) Phospholipid scramblase 3 controls mitochondrial structure, function, and apoptotic response. Mol Cancer Res 1:892-902.

Liu J, Epand RF, Durrant D, Grossman D, Chi NW, Epand RM, Lee RM (2008) Role of phospholipid scramblase 3 in the regulation of tumor necrosis factor-alpha-induced apoptosis. Biochemistry 47:4518-4529.

Liu Y, Levine B (2015) Autosis and autophagic cell death: the dark side of autophagy. Cell Death Differ 22:367-376.

Liu Y, Wang R, Zhao Z, Dong W, Zhang X, Chen X, Ma L (2017a) Shortterm caloric restriction exerts neuroprotective effects following mild traumatic brain injury by promoting autophagy and inhibiting astrocyte activation. Behav Brain Res 331:135-142.

Liu Y, Bao Z, Xu X, Chao H, Lin C, Li Z, Liu Y, Wang X, You Y, Liu N, Ji J (2017b) Extracellular signal-regulated Kinase/Nuclear factor-Erythroid2-like2/Heme oxygenase-1 pathway-mediated mitophagy alleviates traumatic brain injury-induced intestinal mucosa damage and epithelial barrier dysfunction. J Neurotrauma 34:2119-2131.

Luo CL, Li BX, Li QQ, Chen XP, Sun YX, Bao HJ, Dai DK, Shen YW, Xu HF, Ni H, Wan L, Qin ZH, Tao LY, Zhao ZQ (2011) Autophagy is involved in traumatic brain injury-induced cell death and contributes to functional outcome deficits in mice. Neuroscience 184:54-63.

Mukhida K, Kobayashi NR, Mendez I (2005) A novel role for parkin in trauma-induced central nervous system secondary injury. Med Hypotheses 64:1120-1123.

Osier ND, Dixon CE (2016) The controlled cortical impact model: applications, considerations for researchers, and future directions. Front Neurol 7:134.

Park E, Bell JD, Baker AJ (2008) Traumatic brain injury: can the consequences be stopped? CMAJ 178:1163-1170.

Pickrell AM, Youle RJ (2015) The roles of PINK1, parkin, and mitochondrial fidelity in Parkinson's disease. Neuron 85:257-273.

Preau S, Delguste F, Yu Y, Remy-Jouet I, Richard V, Saulnier F, Boulanger E, Neviere R (2016) Endotoxemia engages the RhoA kinase pathway to impair cardiac function by altering cytoskeleton, mitochondrial fission, and autophagy. Antioxid Redox Signal 24:529-542.

Qian W, Wang J, Van Houten B (2013) The role of dynamin-related protein 
1 in cancer growth: a promising therapeutic target? Expert Opin Ther Targets 17:997-1001.

Rosdah AA, K Holien J, Delbridge LM, Dusting GJ, Lim SY (2016) Mitochondrial fission: a drug target for cytoprotection or cytodestruction? Pharmacol Res Perspect 4:e00235.

Sarkar C, Zhao Z, Aungst S, Sabirzhanov B, Faden AI, Lipinski MM (2014) Impaired autophagy flux is associated with neuronal cell death after traumatic brain injury. Autophagy 10:2208-2222.

Scafidi S, Racz J, Hazelton J, McKenna MC, Fiskum G (2010) Neuroprotection by acetyl-L-carnitine after traumatic injury to the immature rat brain. Dev Neurosci 32:480-487.

Schneider CA, Rasband WS, Eliceiri KW (2012) NIH image to ImageJ: 25 years of image analysis. Nat Methods 9:671-675.

Shellington DK, Du L, Wu X, Exo J, Vagni V, Ma L, Janesko-Feldman K, Clark RS, Bayir H, Dixon CE, Jenkins LW, Hsia CJ, Kochanek PM (2011) Polynitroxylated pegylated hemoglobin: a novel neuroprotective hemoglobin for acute volume-limited fluid resuscitation after combined traumatic brain injury and hemorrhagic hypotension in mice. Crit Care Med 39:494-505.

Smith CM, Chen Y, Sullivan ML, Kochanek PM, Clark RS (2011) Autophagy in acute brain injury: feast, famine, or folly? Neurobiol Dis 43:52-59.

Smith G, Gallo G (2017) To mdivi-1 or not to mdivi-1: is that the question? Dev Neurobiol 77:1260-1268.

Sun Y, Vashisht AA, Tchieu J, Wohlschlegel JA, Dreier L (2012) Voltagedependent anion channels (VDACs) recruit parkin to defective mitochondria to promote mitochondrial autophagy. J Biol Chem 287:40652-40660.

Tang YC, Tian HX, Yi T, Chen HB (2016) The critical roles of mitophagy in cerebral ischemia. Protein Cell 7:699-713.

Twig G, Elorza A, Molina AJ, Mohamed H, Wikstrom JD, Walzer G, Stiles L, Haigh SE, Katz S, Las G, Alroy J, Wu M, Py BF, Yuan J, Deeney JT, Corkey
BE, Shirihai OS (2008) Fission and selective fusion govern mitochondrial segregation and elimination by autophagy. EMBO J 27:433-446.

Wang C, Hu Z, Zou Y, Xiang M, Jiang Y, Botchway BOA, Huo X, Du X, Fang M (2017) The post-therapeutic effect of rapamycin in mild traumatic brain-injured rats ensuing in the upregulation of autophagy and mitophagy. Cell Biol Int 41:1039-1047.

Wang Z, Shi XY, Yin J, Zuo G, Zhang J, Chen G (2012) Role of autophagy in early brain injury after experimental subarachnoid hemorrhage. J Mol Neurosci 46:192-202.

Whalen MJ, Carlos TM, Dixon CE, Schiding JK, Clark RS, Baum E, Yan HQ, Marion DW, Kochanek PM (1999) Effect of traumatic brain injury in mice deficient in intercellular adhesion molecule-1: assessment of histopathologic and functional outcome. J Neurotrauma 16:299-309.

Wu Q, Luo CL, Tao LY (2017) Dynamin-related protein 1 (Drp1) mediating mitophagy contributes to the pathophysiology of nervous system diseases and brain injury. Histol Histopathol 32:551-559.

Wu YT, Tan HL, Shui G, Bauvy C, Huang Q, Wenk MR, Ong CN, Codogno P, Shen HM (2010) Dual role of 3-methyladenine in modulation of autophagy via different temporal patterns of inhibition on class I and III phosphoinositide 3-kinase. J Biol Chem 285:10850-10861.

Yamashita SI, Jin X, Furukawa K, Hamasaki M, Nezu A, Otera H, Saigusa T, Yoshimori T, Sakai Y, Mihara K, Kanki T (2016) Mitochondrial division occurs concurrently with autophagosome formation but independently of Drp1 during mitophagy. J Cell Biol 215:649-665.

Zhang X, Yan H, Yuan Y, Gao J, Shen Z, Cheng Y, Shen Y, Wang RR, Wang X, Hu WW, Wang G, Chen Z (2013) Cerebral ischemia-reperfusioninduced autophagy protects against neuronal injury by mitochondrial clearance. Autophagy 9:1321-1333.

Zuo W, Zhang S, Xia CY, Guo XF, He WB, Chen NH (2014) Mitochondria autophagy is induced after hypoxic/ischemic stress in a Drp1-dependent manner: the role of inhibition of Drp1 in ischemic brain damage. Neuropharmacology 86:103-115. 